\title{
BFKL pomeron with massive gluons and running coupling
}

\author{
Eugene $\operatorname{Levin}^{a, b}$, Lev $\operatorname{Lipatov}^{c, d}$ and Marat Siddikov ${ }^{a}$ \\ ${ }^{a}$ Departamento de Física, Universidad Técnica Federico Santa María, \\ y Centro Científico - Tecnológico de Valparaíso, Casilla 110-V, Valparaíso, Chile \\ ${ }^{b}$ Department of Particle Physics, School of Physics and Astronomy, Tel Aviv University, Tel Aviv, 69978, Israel \\ ${ }^{c}$ Theoretical Physics Department, Petersburg Nuclear Physics Institute, \\ Orlova Roscha, Gatchina, 188300, St. Petersburg, Russia and \\ ${ }^{d}$ Physics Department, St.Petersburg State University, Ulyanovskaya 3, St.Petersburg 198504, Russia
}

\begin{abstract}
In this paper we proceed with the study of the Pomeron spectrum, by solving numerically the BFKL equation with massive gluons and running coupling. The spectrum of Regge singularities is discrete and the leading Pomeron has a considerable dependence on nonperturbative effects, for which we use Higgs mechanism as a model. We cross-checked this result with variational method and confirmed the infrared sensitivity of leading Pomeron. This fact is related to the infrared instability of the BFKL equation in QCD, with a running coupling. The subleading poles have a mild sensitivity to the soft physics, and are well described by known semiclassical methods. We also discuss the dependence on various prescriptions of the running coupling arguments.
\end{abstract}

\section{CONTENTS}

I. Introduction

II. Theoretical framework

A. BFKL equation and running coupling $\bar{\alpha}_{S}\left(k, k^{\prime}\right)$

B. Analytic solutions for massless BFKL case 4

C. Higgs mechanism 5

$\begin{array}{lr}\text { III. Results in a massive theory } & 7\end{array}$

A. Results with running coupling (6) 7

$\begin{array}{lr}\text { 1. Semiclassical approximation } & 7\end{array}$

2. Variational method

3. Spectrum from the lattice 11

B. Results with triumvirate coupling [14]

C. Lattice results \& continuum limit

IV. Conclusions

Acknowledgments

A. Study of eigenvalues in the lattice

B. The contribution of the infrared renormalon 18

\begin{tabular}{ll} 
References & 20. \\
\hline
\end{tabular}

\section{INTRODUCTION}

The BFKL Pomeron 1, 2] is a structural element of all effective theories for high energy QCD. It is a solution to the BFKL evolution equation which sums large $\log \left(\bar{\alpha}_{S} \ln (1 / x)\right)^{n}$ terms in the perturbative QCD approach, and gives the 
scattering amplitude at high energies. This amplitude possesses two fundamental properties: the power-like energy dependence of the scattering amplitude ${ }^{1} A \propto(1 / x)^{\omega_{0}}$, where $\omega_{0}=4 \ln 2 \bar{\alpha}_{S}$, and the growth of sizes of the typical dipoles at high energy. The former feature contradicts the Froissart theorem [4], while the second imposes constraint on the applicability of the perturbative approach, when we approach the confinement region. Both problems have been solved in $\mathrm{CGC} /$ saturation approach ${ }^{2}$ though the large impact parameter $(b)$ dependence of the scattering amplitude still remains an open question.

As it has been discussed in Refs. [5] 2], the scattering amplitude at fixed $b$ in this approach should satisfy the unitarity constraint of being smaller than unity, but since the radius of interaction increases as a power of energy, eventually this leads to violation of the Froissart bound [4. Such power-like behavior of the radius is a direct consequence of the perturbative QCD approach, and stems from large impact parameter behavior of the BFKL Pomeron 1 , 2]. Therefore, we have to find how the confinement of quarks and gluons, will affect the large $b$ behavior of the scattering amplitude. Since we are interested in the behavior of the scattering amplitude at large $b$, where the amplitude is small, the saturation effects can be neglected and one can introduce the non-perturbative corrections directly to the BFKL kernel.

In general, the main problem which one needs to solve, is the influence of the unknown infrared behavior on the BFKL Pomeron. It is known that due to a running QCD coupling in a problem, there appears a dimensional scale, $\Lambda_{Q C D}$. According to previous studies in [2, 9-18, due to the running coupling constant, the spectrum of the problem becomes discrete for positive $\omega$, with infinitely many Regge poles with quantum numbers of Pomeron, but also the kernel has unbound from below continuous spectrum for negative $\omega$.

As it has been shown by one of us [2], the spectrum of these Regge poles depends on the behavior of the scattering amplitude in the confinement region. Since the theory of confinement is still in the development stage, nowadays there are two approaches: phenomenological extraction of nonperturbative effects from the experimental data, or their evaluation in an effective model with built-in confinement. The first approach parametrizes the information on confinement in terms of the nonperturbative infrared phases 16 18, and produces a reasonable description of HERA data on deep inelastic scattering [16 18. However, this analysis is complicated by the fact that due to a limited energy range and not sufficiently small $\langle x\rangle \sim 10^{-3}$ in HERA kinematics, a very large number of poles in a series need to be included in the fit, affecting the precision of the extracted parameters. In this paper we address the problem using the second approach, namely studying the spectrum of the BFKL Pomeron in the particular model of infrared behavior, the non-abelian theory with the Higgs mechanism for the gluon mass generation [19, 20]. This gauge invariant and normalizable model has a correct large- $b$ behavior $\propto \exp (-m b)$ of the scattering amplitude. At short distances $r \ll m$, where $m$ is the effective gluon mass, this model transforms smoothly into perturbative QCD, while at distances $r \sim 1 / m$ the gluon acquires a finite mass, in agreement with what was found for correlation functions from eliminating Gribov's copies [21] (see Refs. 22 24]). We wish to stress that a gauge theory with the Higgs mechanism leads to a good description of the gluon propagator calculated in lattice approach [25], with gluon mass $m=0.54 \mathrm{GeV}$.

The first attempt to find the spectrum of the BFKL Pomeron Higgs model with running $\bar{\alpha}_{S}$, was undertaken in [26] by one of us (see also [12]), in the semiclassical approach. However, the underlying assumptions of the semiclassical approach are not fulfilled for all momenta, and for the leading intercept, this uncertainty might be substantial. For this reason, in this paper we cross-check the results and solve numerically the BFKL equation with a running QCD coupling constant. We study how the infrared behavior of a theory influences its spectrum. Finally, we study the spectrum with the so-called triumvirate form of the running $\alpha_{S}$ [10, 27, 29] used in [2, 9] 18, and demonstrate that it has qualitatively the same spectrum. We expect that similar results are valid for all other forms of running couplings, for example in BLM approach [30, 31].

The paper is structured as follows. In Section II we briefly recapitulate the main properties of the BFKL equation with running coupling, as well as analyze different schemes of infrared regularization. In Section III we present our results, and analyze their dependence on the choice of the regularization scheme, as well as on the low-energy confinement model. We use three different approaches: the semi-classical approximation (based on the method of steepest descent), the variational method (based on exact solution to the BFKL equation in diffusion approximation) and the numerical study of the spectrum in the lattice. Finally, in Section [V] we draw conclusions.

\footnotetext{
1 This behavior is valid for all sizes of interacting colorless dipoles. We refer to the book of Ref. 3] for discussion on why in QCD the colorless dipoles are the correct degrees of freedom at high energy.

${ }^{2}$ See book 3 for the review
} 


\section{THEORETICAL FRAMEWORK}

\section{A. BFKL equation and running coupling $\bar{\alpha}_{S}\left(k, k^{\prime}\right)$}

The BFKL equation with running $\bar{\alpha}_{S}$ can be written as an eigenvalue problem

$$
\omega \Phi_{\omega}(k)=-\hat{\mathcal{H}} \Phi_{\omega}=\int \frac{d^{2} k^{\prime}}{2 \pi} \bar{\alpha}_{S}\left(k, k^{\prime}\right) K\left(k, k^{\prime}\right) \Phi_{\omega}\left(k^{\prime}\right),
$$

where the rapidity $(Y)$ dependent scattering amplitude $\mathcal{A}(Y, k)$, is related to $\phi_{\omega}$ as

$$
\mathcal{A}(Y, k)=\int_{\epsilon-i \infty}^{\epsilon+i \infty} \frac{d \omega}{2 \pi i} e^{\omega Y} \phi_{\omega}(k)
$$

and the kernel $K$ is defined as [1, 2]

$$
\int d^{2} k^{\prime} \bar{\alpha}_{S}\left(k, k^{\prime}\right) K\left(k, k^{\prime}\right) \phi_{\omega}\left(k^{\prime}\right)=\int d^{2} k^{\prime} \bar{\alpha}_{S}\left(k, k^{\prime}\right)\left[\frac{\phi_{\omega}\left(k^{\prime}\right)}{\left(\vec{k}-\vec{k}^{\prime}\right)^{2}}-\frac{k^{2} \phi_{\omega}(k)}{\left(\vec{k}-\vec{k}^{\prime}\right)^{2}\left(k^{2}+\left(\vec{k}-\vec{k}^{\prime}\right)^{2}\right)}\right]
$$

For $\bar{\alpha}_{S}(k)=$ const, the conformal symmetry of the kernel $(3)$ allows us to find the eigenfunction of the BFKL equation, in the following form

$$
\phi_{\omega}(k)=\left(k^{2}\right)^{\gamma-1} \equiv e^{(\gamma-1) t} \equiv\left(k^{2}\right)^{-\frac{1}{2} \pm i \nu} \equiv e^{\left(-\frac{1}{2} \pm i \nu\right) t},
$$

where $t=\ln \left(k^{2} / \Lambda_{Q C D}^{2}\right)$ and $\gamma=\frac{1}{2} \pm i \nu$ is a continuous parameter. It is related to eigenvalues as

$$
\omega(\nu)=\bar{\alpha}_{S} \chi(\nu)=\bar{\alpha}_{S}\left(2 \psi(1)-\psi\left(\frac{1}{2}+i \nu\right)-\psi\left(\frac{1}{2}-i \nu\right)\right),
$$

where $\psi(z)$ is the digamma function. The choice of the argument of the running coupling is ambiguous [2, 1117, 26] and is usually done absorbing parts of logarithmic NLO corrections into a definition of $\alpha_{s}$. The simplest form suggested in [26] is

$$
\bar{\alpha}_{S}\left(k, k^{\prime}\right)=\bar{\alpha}_{S}^{\mathrm{LO}}(k)
$$

where $\bar{\alpha}_{\mathrm{LO}}$ is the leading order running coupling in pQCD. The virtue of 6 is that it allows one to evaluate the spectrum and wave functions using approximate semiclassical methods. Although in [26] it was considered for a fixed flavor number scheme, in this paper we use a realistic leading order coupling with a variable flavor number scheme [32]

$$
\begin{aligned}
\bar{\alpha}_{\mathrm{LO}}(k) & =\frac{1}{\beta_{0}^{\text {light }} \ln \left(k^{2} / \Lambda_{\mathrm{QCD}}^{2}\right)-\frac{1}{6} \sum_{i} F\left(k, m_{i}\right)}, \\
\beta_{0}^{\text {light }} & =\frac{11 N_{c}-2 N_{f}^{\text {light }}}{12},
\end{aligned}
$$

where $N_{c}$ is the number of colors, $N_{f}^{\text {light }}$ is the number of light quark flavors, a value of $\Lambda_{Q C D} \approx 150 \mathrm{MeV}$ was fixed from $\alpha_{s}\left(M_{Z}\right) \approx 0.118$. The sum over $i$ in denominator of 7 runs over heavy flavors $c, b, t$, and the threshold function $F$ is given by 32

$$
F\left(k, m_{i}\right) \approx \ln \left(\frac{k^{2}+5 m_{i}^{2}}{\Lambda_{\mathrm{QCD}}^{2}+5 m_{i}^{2}}\right)
$$


As was shown in 33, the form (6) allows one to rewrite the NLO corrections to the BFKL equation, in a form similar to $(3)$, provided we replace the kernel $K$ as

$$
\begin{aligned}
& K\left(k, k^{\prime}\right)=\bar{\alpha}_{S}(k) K^{\mathrm{LO}}\left(k, k^{\prime}\right)+\bar{\alpha}_{S}^{2}(k) K^{\mathrm{NLO}}\left(k, k^{\prime}\right), \\
& \int d^{2} k^{\prime} K^{\mathrm{LO}}\left(k, k^{\prime}\right)\left(k^{2}\right)^{\gamma-1}=\chi(\gamma)\left(k^{2}\right)^{\gamma-1}, \\
& \int d^{2} k^{\prime} K^{\mathrm{NLO}}\left(k, k^{\prime}\right)\left(k^{\prime 2}\right)^{\gamma-1}=\delta(\gamma)\left(k^{2}\right)^{\gamma-1},
\end{aligned}
$$

where $\chi(\gamma)$ is defined in (5), and an explicit form of $\delta(\gamma)$ and $K^{\mathrm{NLO}}\left(k, k^{\prime}\right)$ may be found in [33]. A symmetrized form of Eq. 10, was suggested in [33],

$$
\omega \phi_{\omega}(k)=\int \frac{d^{2} k^{\prime}}{2 \pi} \sqrt{\bar{\alpha}_{S}(k) \bar{\alpha}_{S}\left(k^{\prime}\right)} K^{\mathrm{LO}}\left(k, k^{\prime}\right) \phi_{\omega}\left(k^{\prime}\right),
$$

however, it can be reduced to $(6)$ by redefinition of the wave function $\phi_{\omega}(k)=\sqrt{\bar{\alpha}_{S}(k)} \tilde{\phi}_{\omega}$. The properties of this equation with

$$
\bar{\alpha}_{S}\left(k, k^{\prime}\right)=\bar{\alpha}_{\mathrm{LO}}^{\mathrm{FFNS}}(k)=\frac{1}{\beta_{0}^{\text {light }} \ln \left(k^{2} / \Lambda_{\mathrm{QCD}}\right)}
$$

have been investigated in detail in [2, 11-17] using the semiclassical approximation. A more complicated form of running coupling was suggested in [10, 27,29] and used in [2, 9, 18],

$$
\bar{\alpha}_{S}\left(k, k^{\prime}\right)=\frac{\bar{\alpha}_{\mathrm{LO}}\left(\vec{k}-\vec{k}^{\prime}\right) \bar{\alpha}_{\mathrm{LO}}\left(k^{\prime}\right)}{\bar{\alpha}_{\mathrm{LO}}(k)} .
$$

The form of the QCD coupling given by 14 is preferable, compared to $(13)$ because of the following features:

- Gluon reggeization: It was proven that Eq. (14), rewritten for the octet $t$-channel state and for momentum transferred $q_{T} \neq 0$, satisfies the bootstrap equation [10, 27], i.e. leads to the gluon reggeization as expected on general grounds (see Refs. [28, 29]).

- Summation of Feynman diagrams for large number of flavors $N_{f} \gg 1$ : A direct sum of the Feynman diagrams in the limit of large number of fermions $N_{f}$ leads to the triumvirate structure both for octet and for singlet exchanges in $t$-channel [10, 28, 29]. Due to renormalizability of QCD, this implies that the triumvirate structure is preserved in the general case.

- Correspondence to NLO BFKL equation: In Ref. 34] it is shown that the triumvirate reproduces the term proportional to $\beta_{0}$ in the NLO BFKL kernel.

After renormalization of the fields $\phi_{\omega}(k) \rightarrow \alpha_{s}(k) \phi_{\omega}(k)$, the coupling 14 in front of the BFKL kernel effectively reduces to a function of a difference $\bar{\alpha}_{\mathrm{LO}}\left(\vec{k}-\vec{k}^{\prime}\right)$. However, as we will see below in subsection II C), such transformation does not work for the Regge trajectory 23 with $m_{H}=0$.

\section{B. Analytic solutions for massless BFKL case}

A general analysis of spectrum with a coupling constant (147) is quite complicated, so in the pioneering papers 2 , 10 14 a BFKL spectrum was analyzed with $\bar{\alpha}_{S}\left(k, k^{\prime}\right)$, given by Eq. (13) in a simplified fixed-flavor number scheme (FFNS). In this case we can solve Eq. (1) analytically in a Mellin space, making a transform for $\phi_{\omega}(k)$,

$$
\phi_{\omega}(k)=\int_{-\infty}^{\infty} \frac{d \nu}{2 \pi} \tilde{\phi}_{\omega}(\nu) e^{\left(-\frac{1}{2}+i \nu\right) t}
$$


The Eq. 11) for the Mellin image $\tilde{\phi}_{\omega}(\nu)$ becomes [9, 14]

$$
i \beta_{0} \omega \frac{d \tilde{\phi}_{\omega}(\nu)}{d \nu}=\chi(\nu) \tilde{\phi}_{\omega}(\nu)
$$

which has solutions

$$
\tilde{\phi}_{\omega}(\nu)=e^{-\frac{i}{\beta_{0} \omega} \int_{0}^{\nu} \chi\left(\nu^{\prime}\right) d \nu^{\prime}} .
$$

In general (17) is defined up to a real phase. The inverse Mellin transform yields for the BFKL wave function

$$
\begin{aligned}
\phi_{\omega}(t) & =\int_{-\infty}^{\infty} \frac{d \nu}{2 \pi} e^{-\frac{i}{\beta_{0} \omega} \int_{0}^{\nu} \chi\left(\nu^{\prime}\right) d \nu^{\prime}+\left(-\frac{1}{2}+i \nu\right) t} \\
& =\int_{-\infty}^{\infty} \frac{d \nu}{2 \pi}\left(\frac{\Gamma\left(\frac{1}{2}+i \nu\right)}{\Gamma\left(\frac{1}{2}-i \nu\right)} e^{-2 i \psi(1) \nu}\right)^{\frac{1}{\beta_{0} \omega}} e^{\left(-\frac{1}{2}+i \nu\right) t}=\int_{-\infty}^{\infty} \frac{d \nu}{2 \pi} e^{\left(-\frac{1}{2}+i \nu\right) t+i \varphi^{\text {pert }}(\omega, \nu)} \\
\varphi^{\text {pert }}(\omega, \nu) & \equiv \frac{1}{\beta_{0} \omega}\left(\operatorname{Arg}\left[\frac{\Gamma\left(\frac{1}{2}+i \nu\right)}{\Gamma\left(\frac{1}{2}-i \nu\right)}\right]-2 \psi(1) \nu\right)
\end{aligned}
$$

The formal solution (18) should be supplemented with boundary conditions. The first boundary condition stems from the behavior of the wave function at large $t$. As has been shown in [2, 9, in this kinematic region the solution of the BFKL equation should match the solution for the DGLAP evolution equation[35] in double log approximation. In terms of the wave function this means that at positive $\omega$, values of $\nu$ should be real. The second boundary condition comes from the non-perturbative QCD approach, which gives the wave function with the phase $\varphi^{\text {non-pert }}(\omega)$ at fixed value of $t=t_{0}$. This condition for positive $\omega$ results in quantization of the spectrum [2] from the equation

$$
\varphi^{\text {non-pert }}(\omega)=\varphi^{\text {pert }}(\omega, \nu) .
$$

The choice of the value of $t_{0}$ is dictated by a requirement that running QCD coupling $\alpha_{s}$ should be small enough to justify application of the leading order BFKL approach. Since we impose two boundary conditions at $t \rightarrow \infty$ and at $t=t_{0}$, for positive $\omega$ the spectrum should be quantized. For negative $\omega$, we need not impose any conditions at $t \rightarrow \infty$, so the spectrum remains continuous.

In [11, 14, 26] it was found in a semiclassical approach that for large root number $j$ the spectrum is given by

$$
\omega_{j} \approx \frac{0.4085}{j-\frac{1}{4}+\phi^{\text {nonpert }} / \pi-\nu t_{0} / \pi}, \quad j \gg 1 .
$$

However, as we will show below, due to limitations of semiclassical approach the leading pole which controls the high-energy behavior of amplitudes in this scheme has the largest uncertainty. Additionally, there is an uncertainty which stems from the region of small momenta, where perturbative couplings in (1477) become inapplicable due to an infrared pole. This requires some regularization at a low scale.

\section{Higgs mechanism}

The BFKL kernel (3) was obtained in the regime of asymptotically large $|k| \gg \Lambda_{\mathrm{QCD}}$. However it is known that in the regime of small momenta, nonperturbative effects affect drastically all the partons, generating nonperturbative masses [36] and affecting their interactions [37. The non-abelian theory with Higgs mechanism of mass generation is a particular (and a relatively simple) model, in which nonperturbative effects only generate an effective mass and affect the QCD behavior at large distances $r \sim 1 / m$, where $m \approx 540 \mathrm{MeV}$ is the effective gluon mass [21 24]. As was found in [25], this model leads to a good description of the gluon propagator, calculated in lattice approach. In Standard Model [38] this infrared regularization naturally arises in the limit of vanishing Weinberg angle $\theta_{W}=0$. 
The corresponding modification of a kernel (3) in a massive case takes the form (see [19, 20] and Fig. 1)

$$
\omega \phi(k)=\frac{1}{\pi} \underbrace{\int d^{2} k^{\prime} \bar{\alpha}_{S}\left(k, k^{\prime}\right) \frac{\phi\left(k^{\prime}\right)-\frac{1}{2} \frac{k^{2}+m_{H}^{2}}{k^{\prime 2}+m_{H}^{2}} \phi(k)}{\left(k-k^{\prime}\right)^{2}+m_{H}^{2}}}_{\text {kinetic and potential energy terms }}-\underbrace{\frac{N_{c}^{2}+1}{2 \pi N_{c}^{2}} \frac{m_{H}^{2}}{k^{2}+m_{H}^{2}} \int d^{2} k^{\prime} \frac{\alpha_{\mathrm{LO}}^{2}}{\alpha_{\mathrm{LO}}\left(k^{2}\right)} \frac{\phi\left(k^{\prime 2}\right)}{k^{\prime 2}+m_{H}^{2}}}_{\text {contact term }},
$$

where we denoted by $m_{H}$ the effective gluon mass. The coefficient in front of $\phi(k)$ is sometimes referred to as a Regge trajectory

$$
\omega(k)=-\frac{\left(k^{2}+m_{H}^{2}\right)}{2 \pi} \int d^{2} k^{\prime} \frac{\bar{\alpha}_{S}\left(k, k^{\prime}\right)}{\left[\left(k-k^{\prime}\right)^{2}+m_{H}^{2}\right]\left[k^{\prime 2}+m_{H}^{2}\right]} .
$$

For the special case of $(6)$, the equation $(22)$ simplifies to

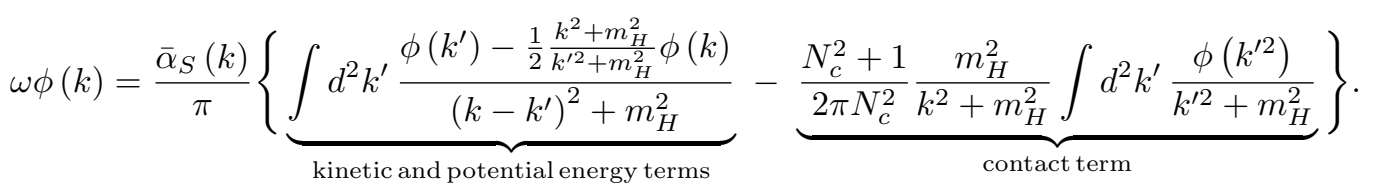

As we can see, both (22) and (24) are not symmetric w.r.t. an interchange of arguments $k$ and $k^{\prime}$, which implies that a kernel operator is no longer hermitian. However, its eigenvalues remain real. Indeed, in the first term of 2224$)$ we can symmetrize the kernel by a redefinition of the wave function $\phi(\kappa) \rightarrow F(\kappa) \phi(\kappa)$, where $F(k)=\alpha(k)$ for $(22)$ with coupling $(14)$, and $F(k)=\sqrt{\alpha(k)}$ for 24 . The contribution of the last (contact) term in 2224 does not affect the eigenvalues [19, 20].

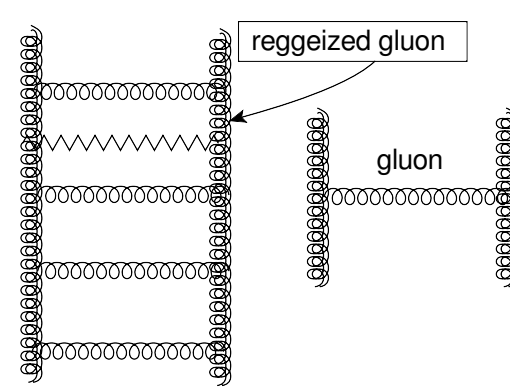

a)

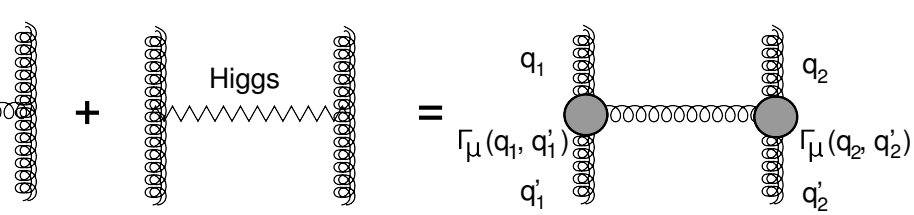

b)

Figure 1. The massive BFKL equation (Fig. 1 1 a)and its kernel (Fig. 1 f b)

The equation (22) has been studied in detail in [19, 20] for the case when $\alpha_{s}\left(k, k^{\prime}\right)=$ const. In this case the integration over the azimuthal angle can be easily done, yielding

$$
\begin{aligned}
& E \phi(\kappa)= \\
& \underbrace{\frac{\kappa+1}{\sqrt{\kappa} \sqrt{\kappa+4}} \ln \frac{\sqrt{\kappa+4}+\sqrt{\kappa}}{\sqrt{\kappa+4}-\sqrt{\kappa}} \phi(\kappa)}_{\text {kinetic energy term }}-\underbrace{\int_{0}^{\infty} \frac{d \kappa^{\prime} \phi\left(\kappa^{\prime}\right)}{\sqrt{\left(\kappa-\kappa^{\prime}\right)^{2}+2\left(\kappa+\kappa^{\prime}\right)+1}}}_{\text {potential energy term }}+\underbrace{\frac{N_{c}^{2}+1}{2 N_{c}^{2} \frac{1}{\kappa+1} \int_{0}^{\infty} \frac{\phi\left(\kappa^{\prime}\right) d \kappa^{\prime}}{\kappa^{\prime}+1}},}_{\text {contact term }},
\end{aligned}
$$

where we introduced dimensionless variables and parameters

$$
\kappa=\frac{k^{2}}{m_{H}^{2}} ; \quad \kappa^{\prime}=\frac{k^{2}}{m_{H}^{2}} ; \quad E=-\frac{\omega}{\bar{\alpha}_{S}} ; \quad \bar{\alpha}_{S}=\frac{\alpha_{S} N_{c}}{\pi} .
$$


It was found that the inclusion of a gluon mass does not affect the eigenvalues of a problem, though changes the wave functions at small momenta. Solving the problem on the lattice (see $\sqrt[A]{ }$ for details), we found that for positive intercepts in a wide range of $\kappa$, the wave functions may be approximated as

$$
\phi_{n}^{(\text {approx })}(\kappa) \approx \frac{\text { const }}{\sqrt{\kappa+4}} \sin (\beta(n) \operatorname{Ln}(\kappa)+\phi(n))
$$

where we introduced the notation

$$
\operatorname{Ln}(\kappa)=\ln \left(\frac{\sqrt{\kappa+4}+\sqrt{\kappa}}{\sqrt{\kappa+4}-\sqrt{\kappa}}\right)
$$

and $\beta(n), \varphi(n)$ are linear functions of a parameter $n, \beta(n)=b n, \varphi(n) \approx b_{\phi} \beta(n)$. The found value of a parameter $b_{\phi}$ does not depend on the lattice choice and is given by $b_{\phi} \approx 1.87$, but the parameter $b$ depends on the lattice size,

$$
b=\frac{2.9}{\ln \left(k_{\max }^{2} /\left(k_{\min }^{2}+m_{H}^{2}\right)\right)} .
$$

In a continuum limit, a root number $n$ is proportional to a parameter $\nu$ in (45), and (27) takes the form

$$
\phi^{\text {(approx) }}(\kappa, \nu)=\frac{\alpha(\nu)}{\sqrt{\kappa+4}} \sin \left(\nu \operatorname{Ln}(\kappa)+b_{\phi} \nu\right) .
$$

The approximation 30 works for all $\nu$, for which $\chi(\nu)$ remains positive. The solution 30 is odd, under $\nu \rightarrow-\nu$. Therefore, our eigenvalues are not degenerate and we have one eigenfunction for each $\nu$.

\section{RESULTS IN A MASSIVE THEORY}

\section{A. Results with running coupling (6)}

In this Section, we will analyze the influence of the confinement region on the eigenvalues of the BFKL equation with running $\bar{\alpha}_{s}$. In order to avoid an infrared pole, we assume that at $t<t_{0}$ the coupling constant is frozen (so the dynamics is described by (25), whereas for $t \gg t_{0}$ we expect that the theory should reacquire the solution (18). Below we compare results for spectra found with two methods, a semiclassical consideration and a lattice result.

\section{Semiclassical approximation}

As was discussed in Section II A, the analytic solution of (1) requires nonperturbative phase fixing (20). Due to the different character of wave functions and spectra (discrete vs continua), the phase matching condition (20) should be supplemented with an additional requirement of equality of eigenvalues

$$
\bar{\alpha}_{S}\left(m_{H}^{2}\right) \chi\left(\nu_{\mathrm{np}}\right)=\omega
$$

where we use a notation $\nu_{\mathrm{np}}$ for the parameter which appears in the non-perturbative regime. The dependence of $\nu_{\mathrm{np}}$ on $\omega$ which follows from 31 is shown in Fig. 2 .

In our approach, we fix the nonperturbative phase from a solution 27). The choice of the matching point $t_{0}$ is arbitrary, provided the running coupling $\alpha_{s}$ is small enough. Following the notation introduced in previous papers $11-$ 14, we use a variable $t=\ln \left(k^{2} / \Lambda_{Q C D}^{2}\right)$. To evaluate the integral over $\nu$ in 18 , we use the method of steepest descent, as was suggested in [11-14]. Since the coefficient in front of the $\mathcal{O}\left(\left(\nu-\nu_{S P}\right)^{2}\right)$ term in the expansion of $\varphi^{\text {pert }}(\omega, \nu)$ is imaginary for small-t, a contour should be taken parallel to the lines bisecting the first and the second quadrants in the complex plane. The saddle point is found from 


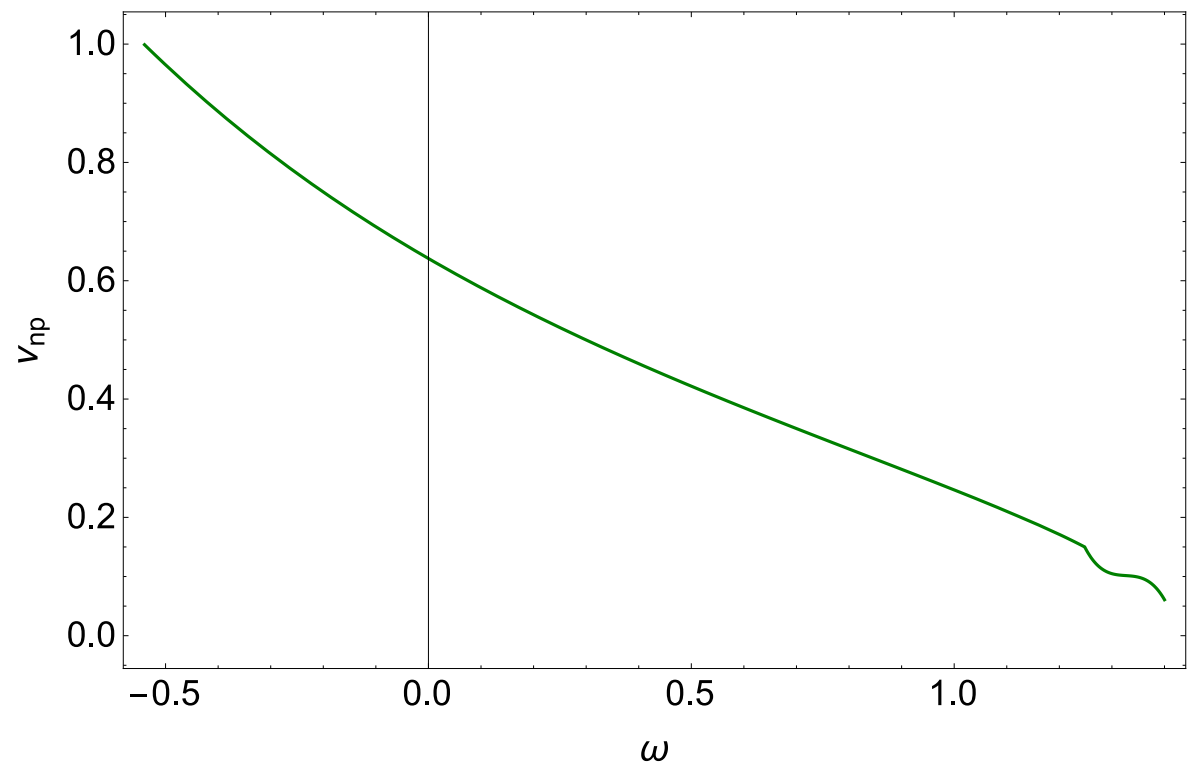

Figure 2. $\quad \nu_{\mathrm{np}}$ versus $\omega$

$$
\xi=\chi\left(\nu_{\mathrm{SP}}\right),
$$

where $\xi=\beta_{0} \omega t$, and we are only interested in roots closest to the real axis. As can be seen from definition of $\chi(\nu)$ in 5 , for real values of $\nu$ this function is restricted from above by value $\sim 4 \ln 2$, which implies that for sufficiently large $\xi$, the relevant saddle-point $\nu_{\mathrm{SP}}$ lies on the imaginary axis (see Fig. 3). In this regime instead of oscillations we have a homogenous decrease with $t$, and a solution corresponds to a DGLAP solution [35] in the double log approximation. As we will see below, the lattice wave functions shown in the Figure 8 , at sufficiently large $t$ confirm this pattern of behavior.

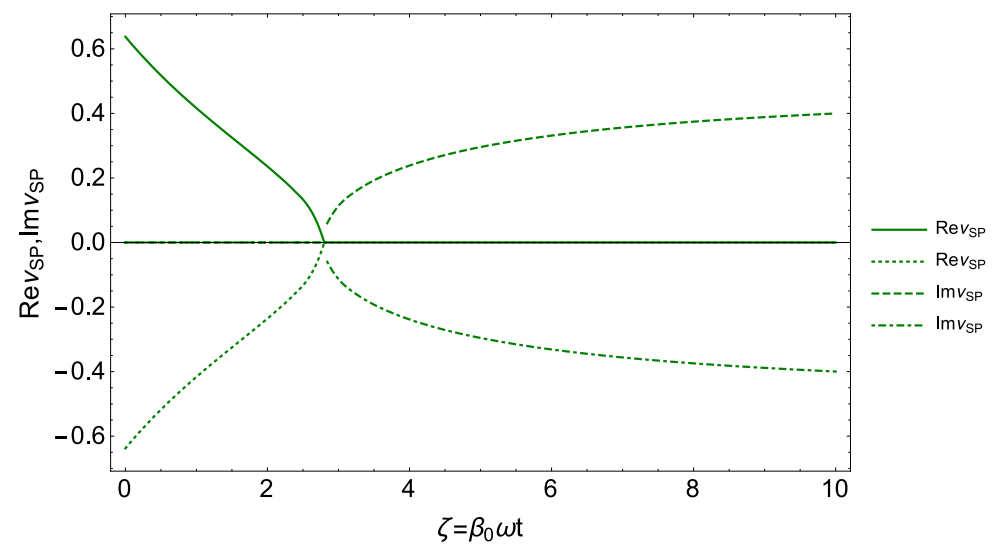

Figure 3. $\operatorname{Re} \nu_{\mathrm{SP}}$ and $\operatorname{Im} \nu_{\mathrm{SP}}$ versus $\xi=\beta_{0} \omega t$ for $\omega>0$. At $\xi \leq \xi_{\text {crit }} \nu_{\mathrm{SP}}$ is real while for $\xi \geq \xi_{\text {crit }}$ is pure imagine.

The phase matching condition 20 in explicit form yields

$$
\varphi^{\text {non-pert }}(\omega)+\pi j=\frac{\pi}{4}+\nu_{S P} t_{0}-2 \frac{\psi(1)}{\beta_{0} \omega} \nu_{S P}+\frac{i}{\beta_{0} \omega} \ln \left(\frac{\Gamma\left(\frac{1}{2}+i \nu_{S P}\right)}{\Gamma\left(\frac{1}{2}-i \nu_{S P}\right)}\right), \quad j \in \mathbb{N} .
$$

The set of Eq. 3233 for positive $\omega$ leads to quantization of spectrum of $\omega$, whereas for negative $\omega$ the spectrum is continuous. Resolving Eq. (33) with respect to $\omega$, we may obtain 


$$
\omega_{j}=\frac{1}{\beta_{0}} \frac{\left\{-2 \psi(1) \nu_{S P}+\operatorname{Im} \ln \left(\frac{\Gamma\left(\frac{1}{2}+i \nu_{S P}\right)}{\Gamma\left(\frac{1}{2}-i \nu_{S P}\right)}\right)\right\}}{\pi\left(j-\frac{1}{4}\right)+\nu_{\mathrm{np}}\left(\omega_{j}\right)\left(\operatorname{Ln}\left(\frac{\Lambda_{Q C D}^{2}}{m_{H}^{2}} e^{t_{0}}\right)+b_{\phi}\right)-\nu_{S P} t_{0}} .
$$

The arbitrariness of the choice of matching point $t_{0}$ can be reinterpreted in terms of uncertainty in the choice of a non-perturbative phase, and would cancel if the $t$-dependence of $\varphi^{\text {pert }}$ and $\varphi^{\text {non-pert }}$ were the same. The dependence of the eigenvalues on the choice of matching point $t_{0}$, is shown in the Fig. 4 . One can see that the sensitivity to the choice of $t_{0}$ is very pronounced for the leading intercept, but decreases rapidly for higher eigenvalues. Such a strong sensitivity to the infrared dynamics (confinement region) can be understood from (18) 3 at asymptotically large momenta the wave functions decrease as $\propto 1 / k^{2}$, while for constant $\bar{\alpha}_{S}$ the asymptotic behavior was $\propto 1 / \sqrt{k^{2}}$. However, due to nodes for higher excited states, the sensitivity to small momenta is diminished, while it remains pronounced for the leading intercept. Similar sensitivity to the infrared was obtained recently in [39]. In what follows for the sake of definiteness we fix $t_{0}$ as $t_{0}=\ln \left(m_{H}^{2} / \Lambda_{\mathrm{QCD}}^{2}\right)$, which reduces 34 ) to

$$
\omega_{j}=\frac{0.4085}{\left(j-\frac{1}{4}\right)+2.827 \nu_{\mathrm{np}}\left(\omega_{j}\right)-1.65}
$$

This result agrees with [26] for $t_{0} \approx 0$.

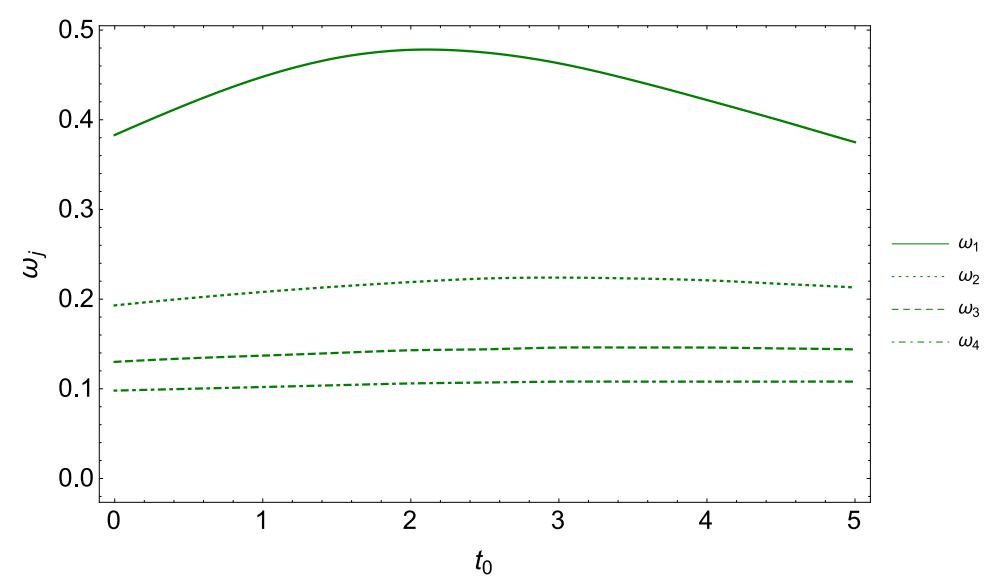

Figure 4. The values of the first four eigenvalues of the BFKL equation in semi-classical approach versus $t_{0}$.

The region of validity of the saddle-point approximation might be estimated from the omitted $\mathcal{O}\left(\left(\nu-\nu_{S P}\right)^{3}\right)$ terms. For the saddle-point integral, the dominant contribution comes from the region $|\delta \nu|<\delta \nu_{\max }$, where

$$
\delta \nu_{\max }=\sqrt{\beta_{0} \omega /\left(\frac{1}{2} \chi\left(\nu_{\mathrm{SP}}\right)\right)} .
$$

A ratio $R$ of the $\mathcal{O}\left(\left(\nu-\nu_{S P}\right)^{3}\right)$-terms to $\mathcal{O}\left(\left(\nu-\nu_{S P}\right)^{2}\right)$-terms in this region is given by

$$
R=\omega /\left(\frac{1}{3 !} \chi_{\nu}\left(\nu_{\mathrm{SP}}\right) \frac{1}{\beta_{0} \omega}\left(\sqrt{\beta_{0} \omega /\left(\frac{1}{2} \chi\left(\nu_{\mathrm{SP}}\right)\right)}\right)^{3}\right)^{2}
$$

\footnotetext{
${ }^{3}$ See the Figure 8 below for the illustration of the wave functions
} 


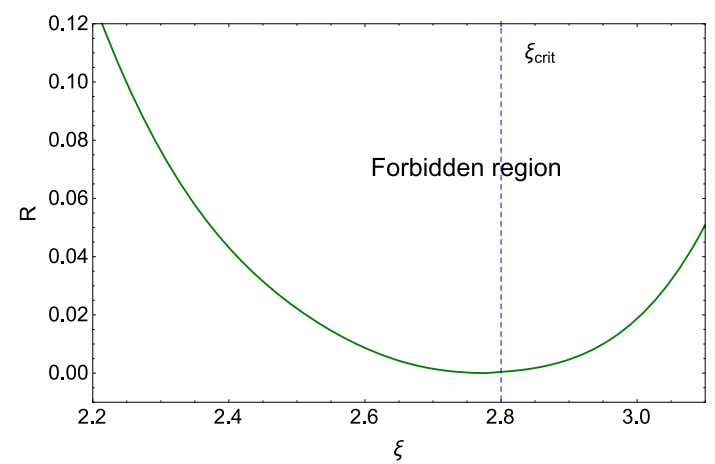

Fig. 5 ra

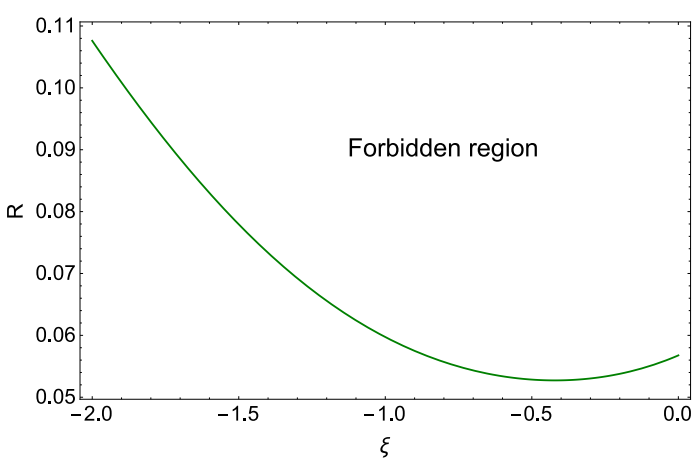

Fig. 5 b

Figure 5. The values of $R$ versus $\xi$ for $\omega>0$ (Fig. 57a)and for $\omega<0$ (Fig. 5.b). For $\omega<R$ we can trust the method of steepest descent.

As we can see from the Fig. 5, this ratio is small for $0<\omega \ll \omega_{\text {crit }}$ and for negative $\omega$, but is not well-justified for the leading eigenvalues, since these wave functions get large contributions from a vicinity of $\xi_{\text {crit }}$.

In the vicinity $\xi \approx \xi_{\text {crit }}$ we can use the diffusion approximation and expand $\chi(\nu)$ as

$$
\chi(\nu) \approx \chi_{0}-D_{0} \nu^{2}+\mathcal{O}\left(\nu^{4}\right)
$$

with $\chi_{0}=4 \ln 2$ and $D_{0}=14 \zeta(3) \approx 16.828$ [1, 3]. Using this approximation, we can take the integral over $\nu^{\prime}$ in Eq. (18) and express it in terms of the Airy functions (see [11 13] for detailed discussions).

\section{Variational method}

In this section we discuss an approximate estimate of the leading eigenvalues using the variational method. This method reduces an integral equation $(25)$ to a minimization problem for the functional

$$
E[\phi]=\frac{\langle\phi|\hat{\mathcal{H}}| \phi\rangle}{\langle\phi \mid \phi\rangle},
$$

where we use a variable $E=-\omega$. The variational method gives an estimate from above for the energy (estimate from below for $\omega_{0}$ ), with precision determined by the fact how close is the chosen trial function to the true eigenfunction. The functional $\langle\phi|\hat{\mathcal{H}}| \phi\rangle$ which corresponds to 11 is given by

$$
\langle\phi|\hat{\mathcal{H}}| \phi\rangle=\int d^{2} k \phi(k) \bar{\alpha}_{S}(k) \int d^{2} k^{\prime}\left[\frac{\phi_{\omega}\left(k^{\prime}\right)}{\left(\vec{k}-\vec{k}^{\prime}\right)^{2}}-\frac{k^{2} \phi_{\omega}(k)}{\left(\vec{k}-\vec{k}^{\prime}\right)^{2}\left(k^{2}+\left(\vec{k}-\vec{k}^{\prime}\right)^{2}\right)}\right] .
$$

Inspired by the solution in the diffusion approximation [10, 11, 13, which is exact in the region of $\omega$ in the vicinity of leading pole, we parametrize our trial function as

$$
t \geq t_{0} \quad \phi^{\text {trial }}(k ; \omega, a)=C(\omega)\left(\frac{t}{e^{t}+a / \Lambda_{\mathrm{QCD}}^{2}}\right)^{1 / 2} \operatorname{Ai}\left[\left(\frac{\omega}{D_{0} \beta_{0}}\right)^{1 / 3}\left(\ln \left(e^{t}+a / \Lambda_{\mathrm{QCD}}^{2}\right)-\frac{\chi_{0}}{\omega}\right)\right],
$$

where $\operatorname{Ai}(x)$ is the Airy function, the parameters $\chi_{0}$ and $D_{0}$ are defined as Taylor expansion coefficients in $(38)$, and for $t<t_{0}$, we use two models of boundary conditions

$$
\begin{aligned}
& \text { model I: } \quad \phi^{\text {trial }}(k ; \omega, a)=0 \\
& \text { model II: } \quad \phi^{\text {trial }}(k ; \omega, a)=\phi^{\text {(approx) }}\left(\kappa, \nu_{\mathrm{np}}\right) .
\end{aligned}
$$


The function $\phi^{\text {(approx) }}\left(\kappa, \nu_{\mathrm{np}}\right)$ in 43 is given by equation 30 and $\nu_{\mathrm{np}}$ is determined by equation 31 .

The model (42) assumes that confinement does not contribute to the BFKL dynamics (so this region can be dropped completely) while the model (43) corresponds to the confinement model discussed in Section II C] The former can be confronted with the numerical solution of the next section (see columns with different $t_{0}$ in the Table I), while the latter we can compare with the semi-classical approach. From Figure (6) we can see that we have maxima in both cases. For model (42) the eigenvalue is equal to 0.5 for $a=0$, and differs from the lattice result (column " $t_{0}=2.5$ ") by $\left(\omega_{\text {exact }}-\omega_{\text {var. med. }}\right) / \omega_{\text {exact }} \approx 16 \%$. The model II leads to $\omega_{0}=-E_{0}=0.42$. Comparing this value with the semiclassical approach we see $\left(\omega_{\mathrm{SCA}}-\omega_{\text {var. med. }}\right) / \omega_{\mathrm{SCA}} \approx 11-12 \%$. However, comparing with the exact solution given by column $\omega_{j}\left(k^{2}+m_{H}^{2}\right)$ we see that $\left(\omega_{\text {exact }}-\omega_{\text {var. med. }}\right) / \omega_{\text {exact }} \approx 5 \%$.

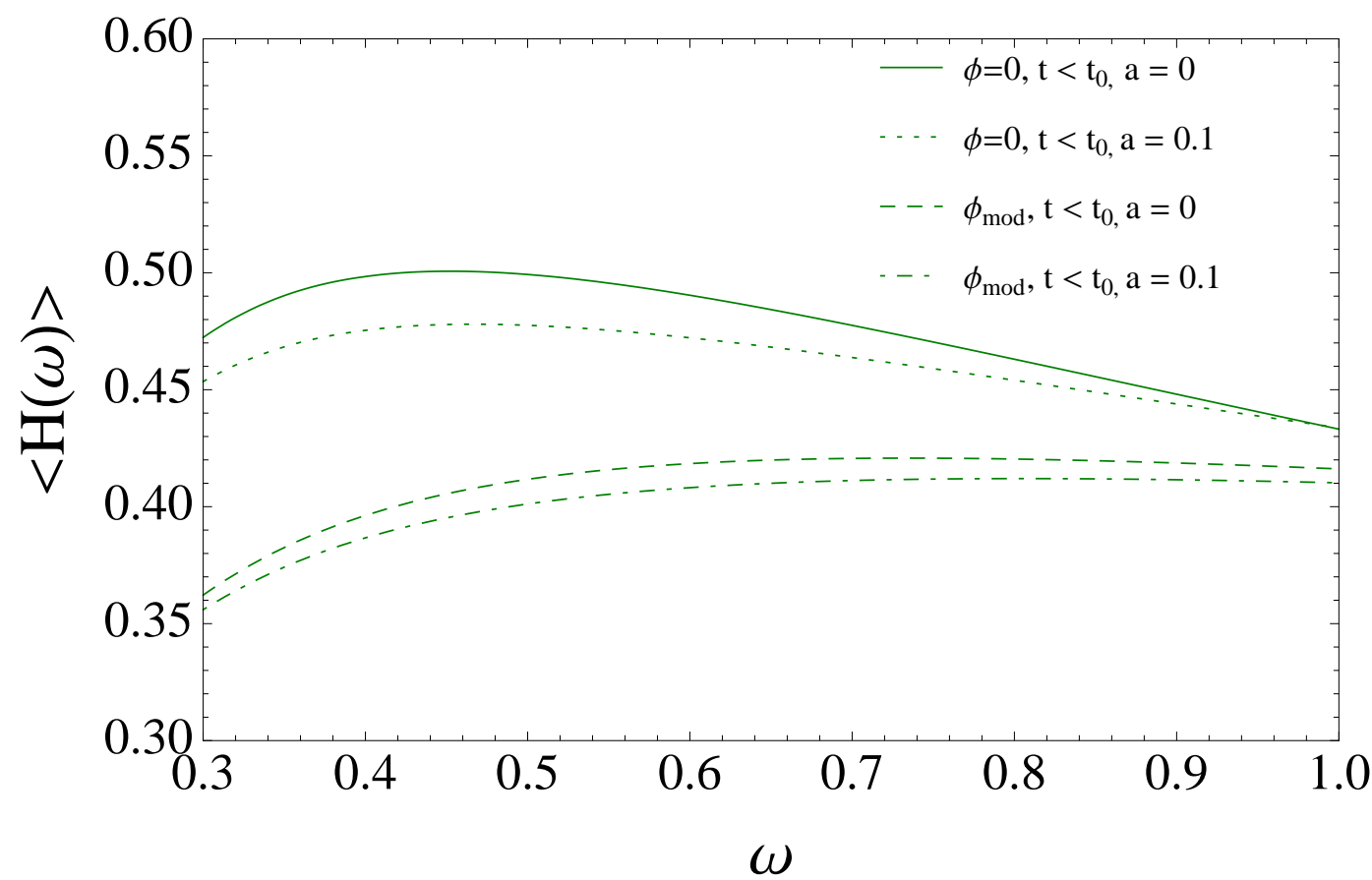

Figure 6. Dependence of $E[\phi]$ given by equation $\left(39 . t_{0}=\ln \left(m_{H}^{2} / \Lambda_{\mathrm{QCD}}\right)=2.5 .\langle H(\omega)\rangle\right.$ denotes the functional of equation $39 . \phi_{\bmod } \equiv \phi^{\text {(approx) }}$.

Also, we analyze spectrum with more general trial function 41 given by

$$
\phi^{\text {trial }}(k ; \omega, a)=\mathcal{N}\left(\frac{\ln \left(k^{2} / \Lambda_{\mathrm{QCD}}^{2}+a^{2}\right)}{k^{2} / \Lambda_{\mathrm{QCD}}^{2}+a^{2}}\right)^{1 / 2} \operatorname{Ai}\left[b\left(\ln \left(k^{2} / \Lambda_{\mathrm{QCD}}^{2}+a^{2}\right)-c\right)\right]
$$

where $a, b, c$ are free parameters. After numerical minimization, the best local minimum which we found corresponds to $a \approx 1.02, b \approx 0.27, c \approx 1.08$, and the leading intercept $\omega \approx 0.58$ is only $2 \%$ below the result found in the lattice. In a similar fashion we may generalize the method and estimate subleading eigenvalues imposing in 39 additional orthogonality conditions $\forall l<j, \quad \int d^{2} k \phi\left(k, \omega_{j}\right) \phi\left(\omega_{l}, k ;\right)=0$, however the precision of such extraction decreases with eigenvalue number $j$ due to accumulation of errors of trial functions.

\section{Spectrum from the lattice}

In order to cross-check our results, we also study the spectrum and eigenfunctions using a lattice method introduced in [19, 20, (see Appendix A] for details). In this method we no longer need to impose a nonperturbative phase fixing (20), and consider instead two alternative schemes of infrared regularization. First, we assume that the gluon has no mass and set a minimal momentum $\kappa_{m i n}=\Lambda_{\mathrm{QCD}}^{2} / m_{H}^{2} \exp \left(t_{0}\right)$. As we can see from the second column of the 


\begin{tabular}{|c|c|c|c|c|c|c|c|c|c|c|c|}
\hline \multirow[t]{2}{*}{$\bar{j}$} & SCA & \multicolumn{4}{|c|}{ Fig (7) } & \multirow[t]{2}{*}{$\omega_{j}\left[\bar{\alpha}_{S}\left(k^{2}+m_{H}^{2}\right)\right.$} & \multicolumn{2}{|c|}{ Triumvirate 4} & \multicolumn{3}{|c|}{ Triumvirate $(46)$} \\
\hline & $t_{0}=2.5$ & $t_{0}=0.1$ & $t_{0}=0.8$ & $t_{0}=1.8$ & $t_{0}=2.5$ & & $\lambda=0.1$ & $\lambda=1$ & $\lambda=0.05$ & $\lambda=0.2$ & $\lambda=1$ \\
\hline 1 & 0.475 & 0.626 & 0.609 & 0.599 & 0.595 & 0.442 & 0.509 & 0.418 & 0.479 & 0.430 & 0.381 \\
\hline 2 & 0.223 & 0.268 & 0.266 & 0.266 & 0.265 & 0.236 & 0.276 & 0.254 & 0.258 & 0.240 & 0.221 \\
\hline 3 & 0.144 & 0.176 & 0.176 & 0.175 & 0.175 & 0.164 & 0.187 & 0.177 & 0.178 & 0.168 & 0.158 \\
\hline 4 & 0.107 & 0.132 & 0.132 & 0.132 & 0.132 & 0.126 & 0.141 & 0.136 & 0.135 & 0.130 & 0.123 \\
\hline 5 & 0.085 & 0.106 & 0.106 & 0.106 & 0.106 & 0.102 & 0.114 & 0.110 & 0.109 & 0.105 & 0.101 \\
\hline 6 & 0.070 & 0.089 & 0.089 & 0.089 & 0.089 & 0.086 & 0.095 & 0.093 & 0.092 & 0.089 & 0.085 \\
\hline 7 & 0.060 & 0.076 & 0.076 & 0.076 & 0.076 & 0.074 & 0.081 & 0.080 & 0.079 & 0.077 & 0.074 \\
\hline 8 & 0.052 & 0.067 & 0.067 & 0.067 & 0.067 & 0.066 & 0.071 & 0.070 & 0.069 & 0.067 & 0.065 \\
\hline 9 & 0.046 & 0.060 & 0.060 & 0.060 & 0.060 & 0.059 & 0.063 & 0.062 & 0.061 & 0.060 & 0.058 \\
\hline 10 & 0.041 & 0.054 & 0.054 & 0.054 & 0.054 & 0.053 & 0.057 & 0.056 & 0.055 & 0.054 & 0.052 \\
\hline
\end{tabular}

Table I. The first ten eigenvalues $\omega_{j}$ of the BFKL equation. First column ("SCA") denotes results with the semiclassical approximation (see Eq. (34)). The second group of columns correspond to eigenvalues with different $t_{0}$ shown in the upper pane of the Figure (7). The column marked by $\omega_{j}\left[\bar{\alpha}_{S}\left(k^{2}+m_{H}^{2}\right)\right]$ is the solution to Eq. 22 with running QCD coupling frozen in low energy domain by shifting variable as $k^{2} \rightarrow k^{2}+M_{H}^{2}$. The group of columns marked as "Triumvirate" corresponds to the solution of 22 with running coupling 45 with two different values of $\lambda$. Finally, the last group of columns corresponds to triumvirate coupling regularized as 46 . In all evaluations we used $M_{H}=540 \mathrm{MeV}$ and $\Lambda_{Q C D}=148 \mathrm{MeV}$.

Table I and upper panel of the Figure (7), the spectrum in this case is discret 4 and has a very weak dependence on $t_{0}$. As expected, for all $t_{0}$ the spectrum lies within uncertainty due to a choice of the nonperturbative phase (light green bands), but the difference with the semiclassical approximation (solid green central line) for the lowest excited states, turns out to be about $20-30 \%$ for the first ten eigenvalues (see columns one and five in Table I). An alternative regularization scheme is to consider (24) in a full range of momenta $k$, freezing the coupling constant in the infrared region by adding some small scale $\lambda m_{H}^{2}$ to its argument. From the lower panel of Figure (7) we can see that the sensitivity to the choice of the scale is also very weak, and agrees with eigenvalues shown in the upper panel. The agreement with the semiclassical approach is quite good, and for the first two eigenvalues, the two approaches agree within 6-7 percent. However, the difference grows for smaller eigenvalues, and reaches 30 percent for the tenth eigenvalue. For the sake of comparison in the same Figure (7) we also plotted eigenvalues of the BFKL equation (25) with fixed coupling $\bar{\alpha}_{s}$. These curves illustrate, that the distance between the eigenvalues due to lattice discretization of continuous spectrum is considerably smaller, and thus the physical spectrum is discrete (see Appendix A for more details).

To summarize, we conclude that results obtained with running coupling (6) are robust with respect to a change of the lower cutoff and freezing scale. For the leading intercept, the sensitivity is largest, but still remains within the phase uncertainty band of semiclassical approximation. At large root number $j$, we observe a significant deviation of the lattice results from semiclassical approximation. This can be understood from (21): when the distance between the roots becomes smaller than the energy discretization due to lattice size, the method can no longer distinguish separate discrete roots and jumps to negative branch (which has continuum spectrum). For this reason for large root number $j$ we should trust the semiclassical approximation.

Finally, in the Figure 8 we plot the wave functions for the first ten eigenvalues and compare them with wave functions evaluated with fixed coupling scheme. As we can see, the falloff at large momenta is much faster than for the case of fixed coupling, in agreement with (18). Also, we note that the nodes are no longer equidistant in the logarithmic coordinates.

We conclude that a running coupling (6) leads to discrete spectrum, however, due to a sensitivity to the choice of the confinement model, the leading intercept is subject to a sizable uncertainty.

\section{B. Results with triumvirate coupling (14)}

In Section $\mathrm{II}$ we discussed that the choice of the coupling (6) is not the only possibility, and the so-called triumvirate parametrization (14) has certain advantages. However, the evaluation of the spectra in semiclassical approximation in this case is impossible, and for this reason, up to now the spectrum of BFKL pomeron with this coupling has

\footnotetext{
${ }^{4}$ In contrast to a discretized spectrum due to finite lattice size as in Eq. 25, the spectrum of 24 is truly discrete because the distance between the neighboring eigenvalues is considerably larger than in case of 25], and does not decrease as a function of upper lattice cutoff $\kappa_{\max }$ (see Section III C and Appendix A for more details).
} 

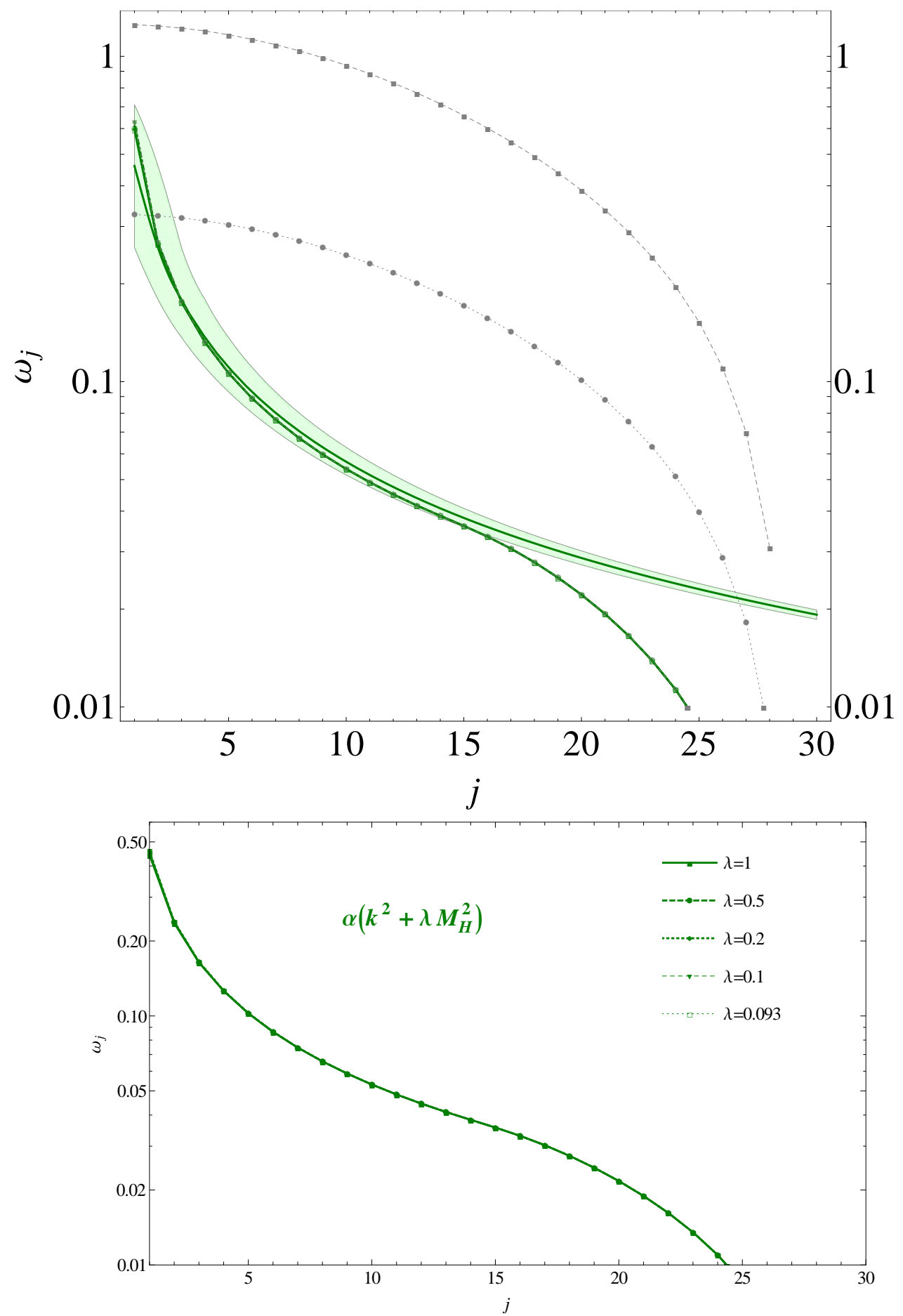

Figure 7. (color online) Upper plot: Dependence of roots $\omega_{j}$ of BFKL on root number $j$ (variable $\nu$ in a continuum limit) for different values of lower cutoff. Light green band corresponds to semiclassical result with the central value evaluated according to (35) and uncertainty of nonperturbative phase $\Delta \varphi= \pm \pi$ (see text for more details). The roots with different values of $t_{0}$ (dark green lines with different dashing patterns, corresponding to $t_{0}$ between 0 and 2.5) cannot be distinguished from the plot; numerical eigenvalues corresponding to different $t_{0}$ may be found in Table 1 For reference we also put thin dashed grey lines which correspond to evaluation with fixed coupling, taken at scales $\alpha_{s}\left(m_{Z}\right)$ and $\alpha_{S}\left(m_{H}\right)$ respectively. Lower plot: Dependence of roots $\omega_{j}$ of BFKL on root number $j$ (variable $\nu$ in a continuum limit) for different schemes of freezing of a coupling constant. As we can see, all curves almost coincide and are not discernible in the plot. 


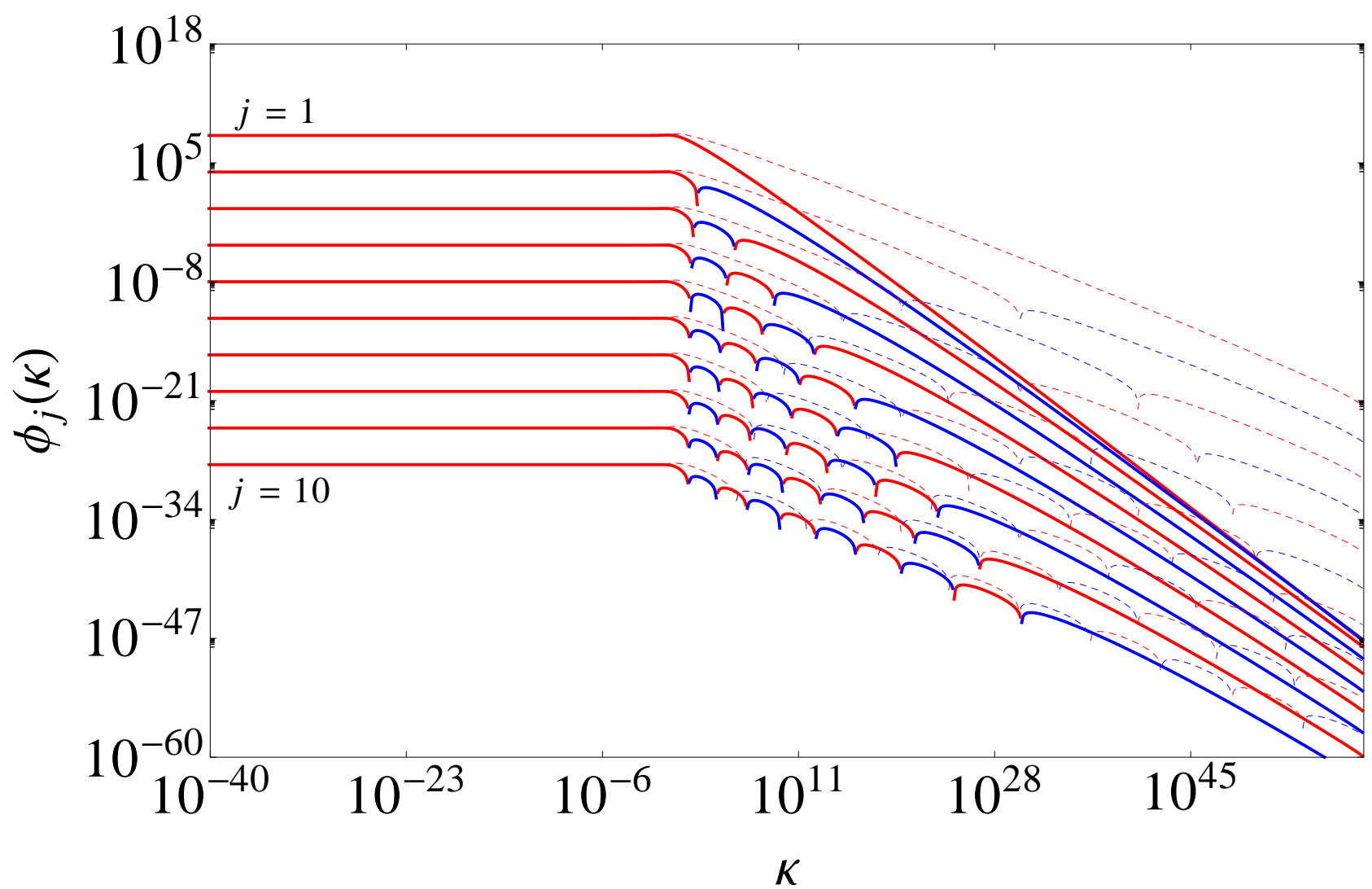

Figure 8. (color online) Wave functions with running coupling which correspond to the first 10 eigenvalues (multiplied by arbitrary constant factor for better visibility). As expected, after ordering by eigenvalues, a number of nodes in the $j$ th wave function equals $j-1$. Changing color (red-blue) reflects change of sign from positive to negative. Thin dashed lines show the results with fixed coupling obtained in our earlier paper [19], normalization chosen to match results with running coupling at small- $\kappa$.

never been studied. In this paper we address this problem, and evaluate the eigenvalues of Eq. 22 using the lattice approach described in Appendix A

In order to regularize the infrared pole in the running coupling $\alpha_{s}$, we introduce a freeze-out scale $\lambda m_{H}^{2}$ into its argument,

$$
\alpha_{s}\left(\left(\vec{k}-\vec{k}^{\prime}\right)^{2}\right) \rightarrow \alpha_{s}\left(\left(\vec{k}-\vec{k}^{\prime}\right)^{2}+\lambda m_{H}^{2}\right)
$$

We found that similar to the case of the coupling (6), the spectrum of the problem is discrete, which manifests itself in significant lattice independent distances between neighboring eigenvalues. From Fig. 9 we can see that the sensitivity to the choice of the infrared scale in 45 is quite mild, which is somewhat unexpected, given the fact that the BFKL kernel (first term in the rhs of (22)) is strongly peaked around $k \approx k^{\prime}$. As we demonstrate in Appendix B. this happens because for large $k$ the contribution of this region is suppressed as $\sim \Lambda_{\mathrm{QCD}} / k$ due to cancellations of singularities of kernel $K$ at $k \approx k^{\prime}$, and for this reason there is a mild sensitivity to the choice of freeze-out scale. In contrast to results of previous section, this sensitivity exists for all eigenvalues (not only the leading intercept). However, numerically eigenvalues of (22) and (24) coincide (within 30\%) with each other. This coincidence is due to the above-mentioned fact that the kernel $\sqrt[22]{2}$ is peaked around $k \approx k^{\prime}$, and for this reason we expect that any other prescription for the running coupling argument (like e.g. 30, 31]) should lead to similar results.

Finally, in order to address the uncertainty in the infrared regularization, we also consider another regularization 
scheme (see Appendix B for details)

$$
\alpha_{\mathrm{eff}}\left(k, k^{\prime}\right) \approx \frac{\bar{\alpha}_{S}(k)}{1+2 b \bar{\alpha}_{S}(k) \ln \left(\frac{\left|k^{2}-k^{\prime 2}\right|}{k^{2}}+\lambda\right)},
$$

where $\lambda$ is a small effective parameter. In the Table (I) we give the first few eigenvalues for several values of $\lambda$. As a function of eigenvalue number $j$, it has a behavior very similar to (45) (shown in Figure 9), for this reason for the sake of legibility in the Figure 110 we only show the dependence on $\lambda$. For $\lambda \approx 1$ the corresponding eigenvalues are much smaller than with a scheme (45), since the former roughly corresponds to the latter with an artificially increased infrared freezeout scale $\sim k^{2}$. However, in the limit $\lambda \rightarrow 0$, the difference between the two schemes vanishes.

\section{Lattice results \& continuum limit}

In this paper we considered two different methods to study the spectrum of BFKL, the semiclassical approximation and the lattice method. The former due to limitations imposed by its applicability is not very reliable for the first leading eigenvalues, with accuracy improving considerably for larger eigenvalues. This is especially important because these first roots determine the behavior of the BFKL theory.

On the contrary, the lattice is applicable for the leading intercepts, but, as we can infer from Fig. (79), becomes unreliable for large root number $j \gg 1$, when the size of the lattice becomes insufficient to resolve the distance between neighboring roots. As a consequence, instead of the infinite series (35) of discrete positive eigenvalues, the lattice sees only a finite number of them, jumping to a negative branch (continuous spectrum). However, as we can see from Figure 11, the number of positive eigenvalues increases with increase of $\kappa_{\max }$, and eventually reproduces an infinite series (35). Similar to the case of fixed coupling (25) studied in [19], we observe that at negative $\omega$ there is a plateau in $j$-dependence: the spectrum freezes near a value $\sim \bar{\alpha}_{s}(0) T(0)$, where $\bar{\alpha}_{s}(0)$ is a value at which running coupling freezes due to infrared regularization, and $T(0)$ is a kinetic energy term (first term in (25)). Together, eigenfunctions corresponding to positive and negative eigenvalues form a complete and orthogonal set of functions.

\section{CONCLUSIONS}

In this paper we applied lattice methods for study of the BFKL spectrum with running QCD, and investigated its dependence on the confinement model and change of running coupling prescriptions. As a model of low-energy (confinement) region we used the non-abelian theory with the Higgs mechanism of mass generation for modeling the behavior of the wave function at large distances. In Section III A we demonstrated that a dependence on the low energy model is weak for all eigenvalues except the leading Regge pole. For the latter, we observed a sizable $(\sim 20-$ $30 \%)$ sensitivity to details of confinement physics. We established that semiclassical result for the leading intercept within uncertainty of the phas $4^{5}$ coincides with exact lattice result, thus justifying application of the semiclassical approach. Also, we estimated the leading eigenvalues with variational method. We used the trial functions based on diffusion approximation solutions of the BFKL equation and demonstrated that this method yields leading eigenvalues consistent with the exact numerical solutions.

In Section IIIB, for the first time we studied the spectrum with the so-called triumvirate form of the running coupling (14) and found that it is discrete. We found that a mild (up to 15\%) dependence on the choice of the freezing scale exists for any eigenvalue, and stems from $\mathcal{O}\left(\Lambda_{Q C D}^{2} / k^{2}\right)$-contributions of renormalons, as discussed in Appendix B However, numerically, eigenvalues of (22) and (24) coincide with each other within 10 percent..

\section{ACKNOWLEDGMENTS}

We thank our colleagues at Tel-Aviv and UTFSM university for encouraging discussions. Our special thanks go to Asher Gotsman, whose comments and suggestions were very instrumental for our work. L.L. acknowledges

\footnotetext{
5 This phase uncertainty, which stems from the unknown confinement region, is the dominant source of uncertainty in the semiclassical consideration and is significantly larger than other sources of uncertainty discussed in Section (III A 1).
} 

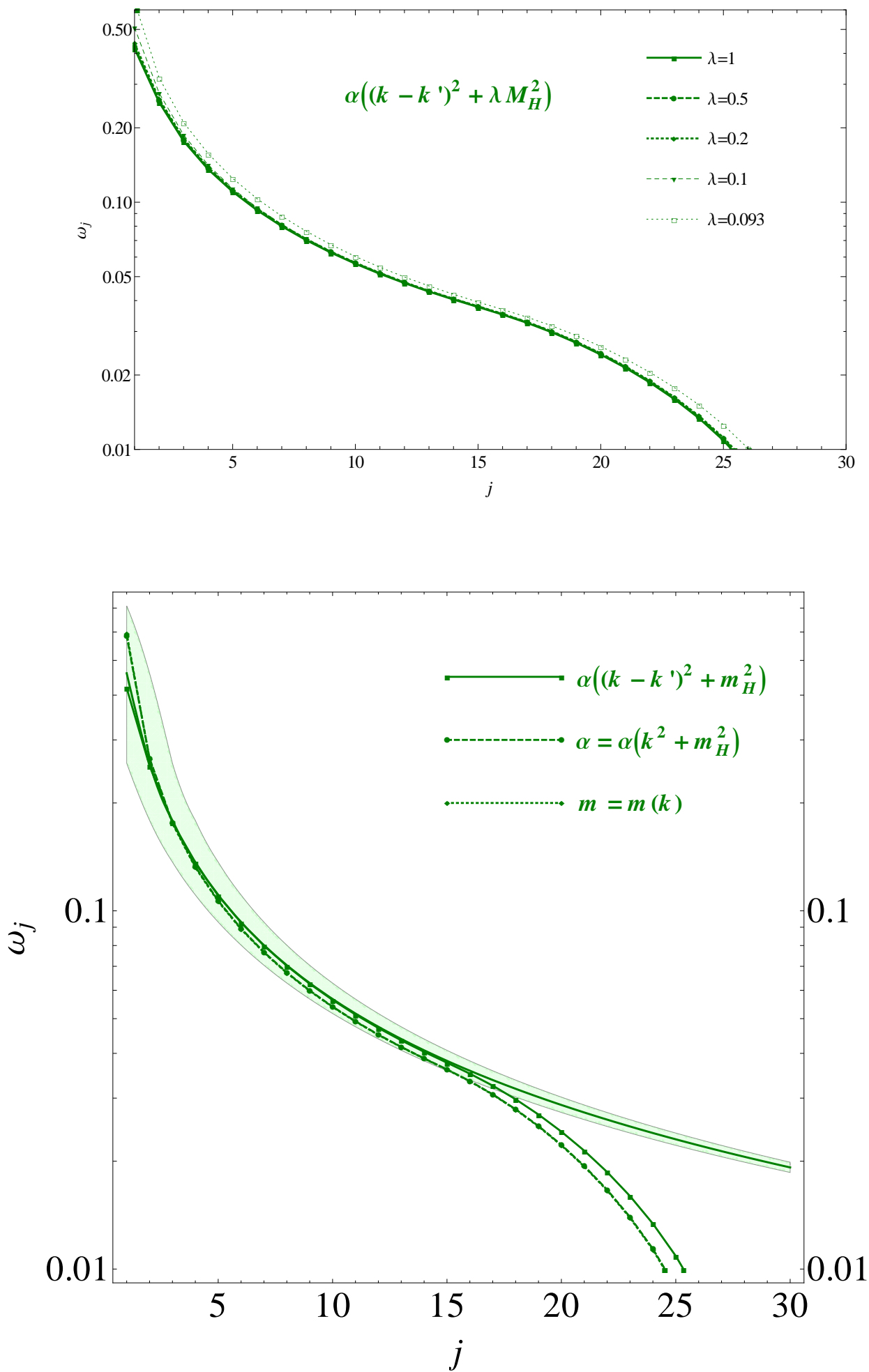

Figure 9. (color online) Upper plot: Eigenvalues of the BFKL equation with a coupling (14). A value of $\lambda$ was introduced to regularize infrared behavior of a coupling constant. Lower plot: A comparison of the eigenvalues evaluated in different IR regularization schemes: adding mass to the argument of a coupling constant with coupling (13) (solid line) and 14) (dashed line), as well as setting the same infrared cutoff as the lowest momentum $k_{\min }$ (dotted line). A value of $\lambda$ was introduced to regularize an infrared behavior of the coupling constant. Light-green band corresponds to a result of semiclassical approximation in (34), with band width reflecting uncertainty due to nonperturbative phase $\varphi^{\text {non-pert }}$; a central line corresponds to a phase $\varphi^{\text {non-pert }}$ set as in 35 . 


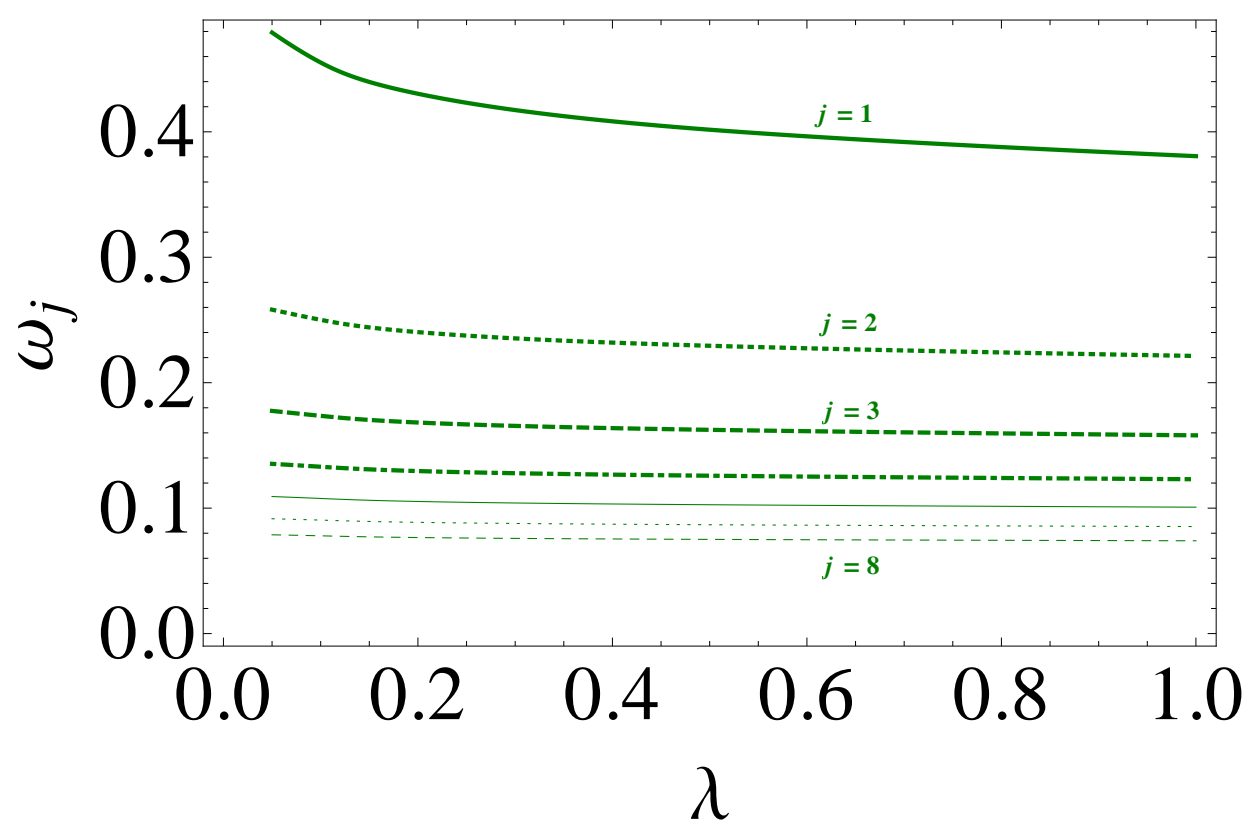

Figure 10. (color online) Dependence of several largest eigenvalues of the BFKL equation with a coupling 46 on a value of infrared parameter $\lambda$.
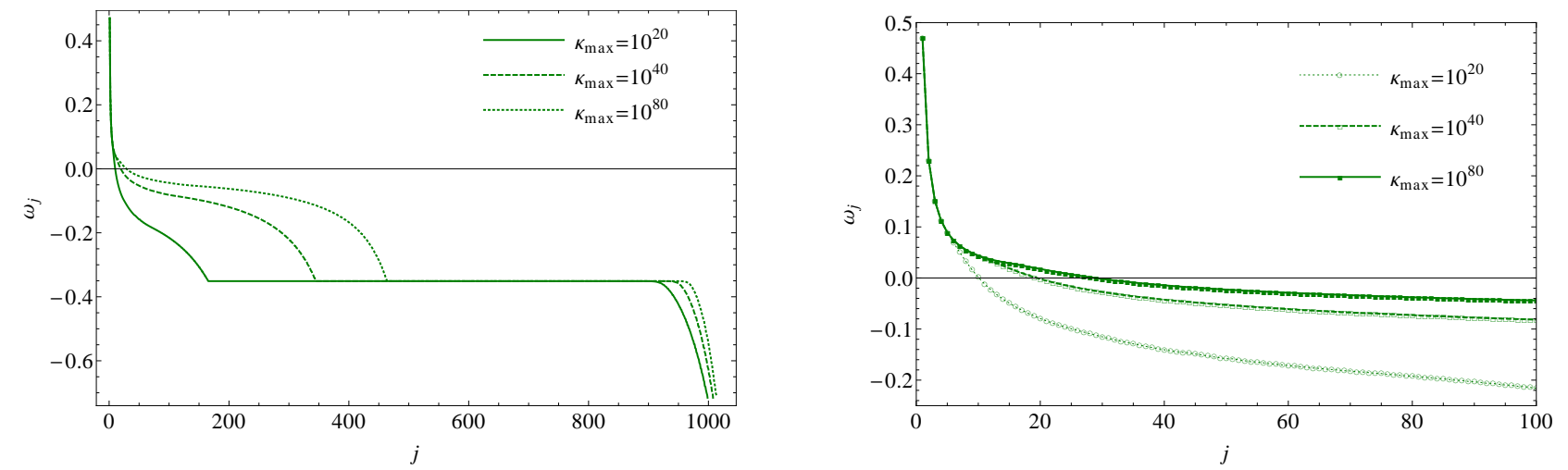

Figure 11. The eigenvalues of the BFKL equation with running coupling 6 versus the values of upper cutoff $\kappa_{\max }$. Right plot is a magnified version of the left plot for small values of $j$.

partial support of the Russian Scientific Fund project 14-2200281. This research was also partially supported by BSF grant 2012124, Proyecto Basal FB 0821 (Chile), the Fondecyt (Chile) grants 1140842 and 1140377, CONICYT (Chile) grants PIA ACT1406 and ACT1413. Powered@NLHPC: This research was partially supported by the supercomputing infrastructure of the NLHPC (ECM-02). Also, we thank Yuri Ivanov for technical support of the USM HPC cluster where part of evaluations were done.

\section{Appendix A: Study of eigenvalues in the lattice}

The equations 2222425 have a common form,

$$
\omega \phi(\kappa)=\int d \kappa^{\prime} K\left(\kappa, \kappa^{\prime}\right) \phi\left(\kappa^{\prime}\right)
$$


where a kernel $K$ is a smooth function of both its arguments and decreases at large momenta as $\sim 1 / \max \left(\kappa, \kappa^{\prime}\right)$. Such large-momenta asymptotics implies that in order to avoid sensitivity to ultraviolet effects, a very large value of the upper cutoff $\kappa_{\max }$ should be introduced. For the numerical calculation of eigenvalue, we replace the continuous variables $\kappa$ and $\kappa^{\prime}$ by the discrete set of $\left\{\kappa_{n}\right\}$ and $\left\{\kappa_{n}^{\prime}\right\}$ using the logarithmic grid (in $\kappa=k^{2} / m^{2}$ ) with $N+1$ nodes,

$$
\kappa_{n}=\kappa_{\min } \exp \left(\frac{n}{N} \ln \left(\kappa_{\max } / \kappa_{\min }\right)\right), \quad n=0, \ldots, N
$$

where the values of $\kappa_{\min }, \kappa_{\max }$ were set to $\kappa_{\min }=10^{-40}, \kappa_{\max }=10^{80}$, and $N=1024$. The equation A1 turns into a discretized linear problem.

As we demonstrated in Ref.[19], for the fixed coupling constant and massless limit, this method reproduces correctly the analytic spectrum (5), with a very mild sensitivity to further improvements of lattice parameters. Due to finite size of the lattice, the spectrum is discretized, with the distance between neighboring eigenvalues $j$ and $j+1$ given by

$$
\Delta \omega_{j+1, j}^{\text {lattice }}=\bar{\alpha}\left\{\left.b \frac{d \chi}{d \nu}\right|_{\nu=b j}+\left.\frac{1}{2} b^{2}(2 j+1) \frac{d^{2} \chi}{d \nu^{2}}\right|_{\nu=b j}\right\}
$$

where parameter $b$ is defined in 29 and vanishes as $\sim 1 / \ln \kappa_{\max }$ in this limit. As we can get from A3, this distance is of order 0.03-0.05 and is considerably smaller than the distance between neighboring eigenvalues for the first leading intercepts $(22 \mid 24)$, which signals that the spectrum is discrete. However, for eigenvalues with larger $j$ a lattice cannot reliably discern neighboring discrete roots.

\section{Appendix B: The contribution of the infrared renormalon}

In this section we discuss the contribution of the infrared renormalon (IR) to the BFKL equation (1) with the triumvirate coupling (14]) (see more in Ref.[10, 40]). For the sake of simplicity we consider the case of massless theory, assuming that the generalization for the case of massive theory is straightforward. Explicitly, the equation (1) can be rewritten in this case as

$$
\omega \phi_{\omega}(k)=\int d^{2} k^{\prime} \frac{\bar{\alpha}_{S}\left(k^{\prime 2}\right)}{\bar{\alpha}_{S}\left(k^{2}\right)} \frac{\bar{\alpha}_{S}\left(\left(\vec{k}-\vec{k}^{\prime}\right)^{2}\right)}{\left(\vec{k}-\vec{k}^{\prime}\right)^{2}}\left\{\phi_{\omega}\left(k^{\prime}\right)-\frac{k^{2}}{k^{\prime 2}+\left(\vec{k}-\vec{k}^{\prime}\right)^{2}} \phi_{\omega}(k)\right\} .
$$

As we can see, a kinematic region $\left(\vec{k}-\vec{k}^{\prime}\right)^{2} \leq \Lambda_{Q C D}^{2}, k \approx k^{\prime} \gg \Lambda_{Q C D}$ can potentially lead to a divergent contribution and thus deserves special attention. In order to integrate over the azimuthal angle $\varphi$, we rewrite a running coupling as

$$
\bar{\alpha}_{S}\left(\left(\vec{k}-\vec{k}^{\prime}\right)^{2}\right)=\frac{\bar{\alpha}_{S}(k)}{1+\beta_{0} \bar{\alpha}_{S}(k) \ln \left(\left(\vec{k}-\vec{k}^{\prime}\right)^{2} / k^{2}\right)}=\bar{\alpha}_{S}\left(k^{2}\right) \sum_{i=0}^{\infty}\left(-\bar{\alpha}_{S}\left(k^{2}\right)\right)^{i} \ln ^{i}\left(\frac{\left(\vec{k}-\vec{k}^{\prime}\right)^{2}}{k^{2}}\right)
$$


After integration of each term over the angle, we obtain ${ }^{6}$

$$
\begin{aligned}
\int d \varphi \frac{\bar{\alpha}_{S}\left(\left(\vec{k}-\vec{k}^{\prime}\right)^{2}\right)}{\left(\vec{k}-\vec{k}^{\prime}\right)^{2}} & =\left.\bar{\alpha}_{S}\left(k^{2}\right) \sum_{i=0}^{\infty}\left(-\bar{\alpha}_{S}\left(k^{2}\right)\right)^{i} \frac{d^{i}}{d \mu^{i}}\right|_{\mu=0} \int \frac{d \varphi\left(k^{2}\right)^{-\mu}}{\left(\left(\vec{k}-\vec{k}^{\prime}\right)^{2}\right)^{1-\mu}} \\
& =\left.\pi \bar{\alpha}_{S}\left(k^{2}\right) \sum_{i=0}^{\infty}\left(-\bar{\alpha}_{S}\left(k^{2}\right)\right)^{i} \frac{d^{i}}{d \mu^{i}}\right|_{\mu=0} \frac{1}{\left|k^{2}-k^{\prime 2}\right|}\left(\frac{\left|k^{2}-k^{\prime 2}\right|}{k^{2}}\right)^{2 \mu}{ }_{2} F_{1}(\mu, \mu, 1, z) \\
& \rightarrow \frac{\pi}{\left|k^{2}-k^{\prime 2}\right|}\left(\frac{\bar{\alpha}_{S}\left(k^{2}\right)}{1+2 \beta_{0} \bar{\alpha}_{S}\left(k^{2}\right) \ln \left(\frac{\left|k^{2}-k^{\prime 2}\right|}{k^{2}}\right)}+\mathcal{O}\left(z \bar{\alpha}_{S}^{3}\left(k^{2}\right)\right)\right),
\end{aligned}
$$

where we introduced $z=k^{\prime 2} / k^{2}$ for $k^{\prime}<k$ and $z=k^{2} / k^{\prime 2}$ for $k^{\prime}>k$. Expanding the term in brackets $\{\ldots\}$ in (B1), we obtain

$$
\begin{aligned}
\omega \phi_{\omega}(k) & =\int d^{2} k^{\prime} \frac{\bar{\alpha}_{S}\left(k^{\prime 2}\right)}{\bar{\alpha}_{S}\left(k^{2}\right)} \frac{\bar{\alpha}_{S}\left(\left(\vec{k}-\vec{k}^{\prime}\right)^{2}\right)}{\left(\vec{k}-\vec{k}^{\prime}\right)^{2}} \\
& \times\left\{\left(1-\frac{k^{2}}{k^{\prime 2}}+\frac{k^{2}\left(\vec{k}-\vec{k}^{\prime}\right)^{2}}{k^{\prime 4}}\right) \phi_{\omega}(k)+\left.\frac{k^{2}-k^{\prime 2}}{k^{2}} \frac{d \phi_{\omega}\left(k^{\prime}\right)}{d \ln \left(k^{\prime 2} / k^{2}\right)}\right|_{k=k^{\prime}}+\ldots\right\}
\end{aligned}
$$

Let us first consider the first term in $\{\ldots\}$ in which we put $\bar{\alpha}_{S}\left(k^{2}\right)=\bar{\alpha}_{S}\left(k^{2}\right)$ in our kinematic region,

$$
\int d^{2} k^{\prime} \frac{\bar{\alpha}_{S}\left(\left(\vec{k}-\vec{k}^{\prime}\right)^{2}\right)}{\left(\vec{k}-\vec{k}^{\prime}\right)^{2}} \frac{k^{\prime 2}-k^{2}}{k^{\prime 2}} \frac{\text { after angle integration }}{\longrightarrow} \int \frac{d k^{\prime 2}}{k^{\prime 2}} \frac{k^{\prime 2}-k^{2}}{\left|k^{\prime 2}-k^{2}\right|} \frac{\bar{\alpha}_{S}(k)}{1+2 b \bar{\alpha}_{S}(k) \ln \left(\frac{\left|k^{2}-k^{\prime 2}\right|}{k^{2}}\right)} .
$$

1. In the region $k^{\prime}>k$ we have

$$
\int \frac{d k^{\prime 2}}{k^{\prime 2}} \frac{k^{\prime 2}-k^{2}}{\left|k^{\prime 2}-k^{2}\right|} \frac{\bar{\alpha}_{S}(k)}{1+2 b \bar{\alpha}_{S}(k) \ln \left(\frac{\left|k^{2}-k^{\prime 2}\right|}{k^{2}}\right)}=\int_{1}^{\infty} d z \frac{\bar{\alpha}_{S}(k)}{1+2 b \bar{\alpha}_{S}(k) \ln (z-1)}
$$

where we introduced $z=k^{\prime 2} / k^{2}$. Although the expansion (B4) is valid only for $k^{\prime} \approx k$, for a moment we will ignore this fact and extend the upper integration limit to $\infty$. Deriving Eq. [B6] we replaced $d k^{\prime 2} / k^{\prime 2} \rightarrow d k^{\prime 2} / k^{2}$. Introducing a new variable $z-1=e^{\frac{1}{2} u}$ we obtain

$$
\int_{1}^{\infty} d z \frac{\bar{\alpha}_{S}(k)}{1+2 b \bar{\alpha}_{S}(k) \ln (z-1)}=\frac{1}{2} \int_{-\infty}^{+\infty} d u e^{\frac{1}{2} u} \frac{\bar{\alpha}_{S}(k)}{1+b \bar{\alpha}_{S}(k) u}
$$

2. In the region $k>k^{\prime}$ we have

$$
\int \frac{d k^{\prime 2}}{k^{\prime 2}} \frac{k^{\prime 2}-k^{2}}{\left|k^{\prime 2}-k^{2}\right|} \frac{\bar{\alpha}_{S}(k)}{1+2 b \bar{\alpha}_{S}(k) \ln \left(\frac{\left|k^{2}-k^{\prime 2}\right|}{k^{2}}\right)}=-\int_{0}^{1} d z \frac{\bar{\alpha}_{S}(k)}{1+2 b \bar{\alpha}_{S}(k) \ln (1-z)}
$$

Introducing $1-z=e^{\frac{1}{2} u}$ we reduce Eq. $\mathrm{B} 8$ to

$$
\frac{1}{2} \int_{0}^{-\infty} d u e^{\frac{1}{2} u} \frac{\bar{\alpha}_{S}(k)}{1+b \bar{\alpha}_{S}(k) u}=-\frac{1}{2} \int_{-\infty}^{0} d u e^{\frac{1}{2} u} \frac{\bar{\alpha}_{S}(k)}{1+b \bar{\alpha}_{S}(k) u}
$$

\footnotetext{
${ }^{6}$ Here we use 3.665(2) and 9.131 from 41]
} 
Summing Eq. (B7) and Eq. (B9) we see that

$$
\int \frac{d k^{\prime 2}}{k^{\prime 2}} \frac{k^{\prime 2}-k^{2}}{\left|k^{\prime 2}-k^{2}\right|} \frac{\bar{\alpha}_{S}(k)}{1+2 b \bar{\alpha}_{S}(k) \ln \left(\frac{\left|k^{2}-k^{\prime 2}\right|}{k^{2}}\right)}=\frac{1}{2} \int_{0}^{+\infty} d u \frac{e^{\frac{1}{2} u}}{1+e^{\frac{1}{2} u}} \frac{\bar{\alpha}_{S}(k)}{1+b \bar{\alpha}_{S}(k) u}
$$

where we replaced $d k^{\prime 2} / k^{2} \rightarrow d k^{\prime 2} / k^{\prime 2}$. Thus, in Eq. B10 the infrared renormalon completely cancels, and only ultraviolet renormalon remains.

In all estimates in this section we neglect the contributions of the order of $\Lambda_{Q C D}^{2} / k^{2}$. We expect that such terms give $\mathcal{O}\left(\Lambda_{Q C D}^{2} / k^{2}\right)$-contribution from the infrared renormalon which are negligible outside of confinement region. The fact that the integral in $(\mathrm{B} 10)$ is only taken over $u>0$ implies that the dominant contribution stems from region $\left(k^{\prime 2}-k^{2}\right) / k^{2}>1$. The last term in the l.h.s. of (B10) can be interpreted as an effective (angular averaged) coupling constant

$$
\alpha_{\mathrm{eff}}\left(k, k^{\prime}\right) \approx \frac{\bar{\alpha}_{S}(k)}{1+2 b \bar{\alpha}_{S}(k) \ln \left(\frac{\left|k^{2}-k^{\prime 2}\right|}{k^{2}}\right)},
$$

which vanishes near the point $k \approx k^{\prime}$. In order to regularize a behavior of (B11) near $k \approx k^{\prime}$, we introduce in the argument of logarithm a small constant $\lambda$, which leads to an effective coupling (46). This form of regularization is inspired by the fact that a relevant parameter in the expansion $\sqrt{\mathrm{B} 4}$ is the ratio $\left(k^{2}-k^{\prime 2}\right) / k^{2}$ rather than $\left(k^{2}-k^{\prime 2}\right) / \Lambda_{\mathrm{QCD}}^{2}$. Besides similar parameter controls the size of higher order corrections in expansion near $k^{\prime} \approx k$ : as can be seen from the structure of the third term in (B4), its relative size is given by

$$
\mathcal{R}=\left.\frac{k^{\prime 2}-k^{2}}{k^{2}} \frac{d \ln \phi_{\omega}\left(k^{\prime}\right)}{d \ln k^{\prime 2}}\right|_{k^{\prime}=k} \approx \lambda \frac{d \ln \phi_{\omega}\left(k^{\prime}\right)}{d \ln k^{\prime 2}}
$$

where $\lambda$ is some parameter. As can be seen from (30), the ratio $d \ln \phi_{\omega}\left(k^{\prime}\right) / d \ln k^{\prime 2} \sim 1$, for this reason $\mathcal{R} \approx \lambda$.

[1] E. A. Kuraev, L. N. Lipatov, and F. S. Fadin, Sov. Phys. JETP 45, 199 (1977); Ya. Ya. Balitsky and L. N. Lipatov, Sov. J. Nucl. Phys. 28, 22 (1978).

[2] L. N. Lipatov, Phys. Rep. 286 (1997) 131; Sov. Phys. JETP 63 (1986) 904 [Zh. Eksp. Teor. Fiz. 90, 1536 (1986)].

[3] Yuri V Kovchegov and Eugene Levin, "Quantum Choromodynamics at High Energies", Cambridge Monographs on Particle Physics, Nuclear Physics and Cosmology, Cambridge University Press, 2012 and references therein.

[4] M. Froissart, Phys. Rev. 123 (1961) 1053;

A. Martin, "Scattering Theory: Unitarity, Analitysity and Crossing." Lecture Notes in Physics, Springer-Verlag, BerlinHeidelberg-New-York, 1969.

[5] A. Kovner and U. A. Wiedemann, Phys. Rev. D 66, 051502 (2002) [hep-ph/0112140].

[6] A. Kovner and U. A. Wiedemann, Phys. Rev. D 66, 034031 (2002) [hep-ph/0204277].

[7] A. Kovner and U. A. Wiedemann, Phys. Lett. B 551, 311 (2003) [hep-ph/0207335].

[8] E. Ferreiro, E. Iancu, K. Itakura and L. McLerran, Nucl. Phys. A 710, 373 (2002) [hep-ph/0206241].

[9] L. V. Gribov, E. M. Levin and M. G. Ryskin, Phys. Rep. 100 (1983) 1.

[10] E. Levin, Nucl. Phys. B 453 (1995) 303 [hep-ph/9412345].

[11] E. Levin, Nucl. Phys. B 545, 481 (1999), hep-ph/9806228.

[12] H. Kowalski, L. N. Lipatov and D. A. Ross, Eur. Phys. J. C 76 (2016) 1, 23, [arXiv:1508.05744 [hep-ph]].

[13] H. Kowalski, L. Lipatov and D. Ross, Eur. Phys. J. C 74 (2014) 6, 2919, [arXiv:1401.6298 [hep-ph]].

[14] H. Kowalski, L. N. Lipatov and D. A. Ross, Phys. Part. Nucl. 44 (2013) 547, arXiv:1205.6713 [hep-ph].

[15] H. Kowalski, L. N. Lipatov and D. A. Ross, "Indirect Evidence for New Physics at the 10 TeV Scale," arXiv:1109.0432 [hep-ph].

[16] H. Kowalski, L. N. Lipatov, D. A. Ross and G. Watt, Nucl. Phys. A 854, 45 (2011); Eur. Phys. J. C 70, 983 (2010) [arXiv:1005.0355 [hep-ph]].

[17] H. Kowalski, L. N. Lipatov, D. A. Ross and G. Watt, Eur. Phys. J. C 70 (2010) 983, [arXiv:1005.0355 [hep-ph]].

[18] E. Levin and I. Potashnikova, JHEP 1402 (2014) 089, [arXiv:1307.7823 [hep-ph]].

[19] E. Levin, L. Lipatov and M. Siddikov, Phys. Rev. D 89 (2014) 7, 074002, [arXiv:1401.4671 [hep-ph]].

[20] E. Levin, L. Lipatov and M. Siddikov, Eur. Phys. J. C 75 (2015) 11, 558, [arXiv:1508.04118 [hep-ph]].

[21] V. N. Gribov, Nucl. Phys. B 139, 1 (1978).

[22] J. Serreau, M. Tissier and A. Tresmontant, Phys. Rev. D 89, 125019 (2014) [arXiv:1307.6019 [hep-th]] J. Serreau and M. Tissier, Phys. Lett. B 712, 97 (2012) [arXiv:1202.3432 [hep-th]]. 
[23] N. Vandersickel and D. Zwanziger, Phys. Rept. 520, 175 (2012) [arXiv:1202.1491 [hep-th]] and references therein.

[24] J. M. Cornwall, Mod. Phys. Lett. A 28, 1330035 (2013) [arXiv:1310.7897 [hep-ph]]; J. A. Gracey, J. Phys. A 47 (2014) 44 , 445401, [arXiv:1409.0455 [hep-ph]].

[25] P. J. Silva, D. Dudal and O. Oliveira, "Spectral densities from the lattice," PoS LATTICE 2013 (2014) 366 [arXiv:1311.3643 [hep-lat]].

P. J. Silva, O. Oliveira, D. Dudal, P. Bicudo and N. Cardoso, b"Many faces of the Landau gauge gluon propagator at zero and finite temperature: positivity violation, spectral density and mass scales," PoS QCD -TNT-III, 040 (2013) [arXiv:1401.1554 [hep-lat]].

[26] L. N. Lipatov, "Non-perturbative effects for the BFKL equation in QCD and in $N=4$ SUSY," AIP Conf. Proc. 1654 (2015) 070004

[27] M. A. Braun, Phys. Lett. B 348 (1995) 190, [hep-ph/9408261].

[28] Y. V. Kovchegov and H. Weigert, Nucl. Phys. A 784 (2007) 188 [hep-ph/0609090].

[29] I. Balitsky, Phys. Rev. D 75 (2007) 014001, [hep-ph/0609105].

[30] S. J. Brodsky, V. S. Fadin, V. T. Kim, L. N. Lipatov and G. B. Pivovarov, JETP Lett. 70, 155 (1999) [hep-ph/9901229].

[31] S. J. Brodsky, V. S. Fadin, V. T. Kim, L. N. Lipatov and G. B. Pivovarov, JETP Lett. 76, 249 (2002) [Pisma Zh. Eksp. Teor. Fiz. 76, 306 (2002)] [hep-ph/0207297].

[32] A. De Rujula and H. Georgi, Phys. Rev. D 13, 1296 (1976).

[33] V. S. Fadin and L. N. Lipatov, Phys. Lett. B 429, 127 (1998) [hep-ph/9802290]; M. Ciafaloni and G. Camici, Phys. Lett. B 430, 349 (1998) [hep-ph/9803389].

[34] Y. V. Kovchegov and H. Weigert, Nucl. Phys. A 789, 260 (2007) [hep-ph/0612071].

[35] V. N. Gribov and L. N. Lipatov, Sov. J. Nucl. Phys 15 (1972) 438;

G. Altarelli and G. Parisi, Nucl. Phys. B 126 (1977) 298;

Yu. l. Dokshitser, Sov. Phys. JETP 46 (1977) 641.

[36] J. M. Cornwall, Phys. Rev. D 26, 1453 (1982).

[37] P. Maris and C. D. Roberts, Int. J. Mod. Phys. E 12, 297 (2003)

[38] J. Bartels, L. N. Lipatov and K. Peters, Nucl. Phys. B 772, 103 (2007) [hep-ph/0610303].

[39] D. A. Ross and A. S. Vera, arXiv:1605.08265 [hep-ph].

[40] E. Gardi, J. Kuokkanen, K. Rummukainen and H. Weigert, Nucl. Phys. A 784 (2007) 282, [hep-ph/0609087].

[41] I. Gradstein and I. Ryzhik, Table of Integrals, Series, and Products, Fifth Edition, Academic Press, London, 1994. [hep-ph/9806482]. 